\title{
A micromechanical basis for partitioning the evolution of grain bridging in brittle materials
}

\author{
J.W. Foulk III ${ }^{\mathrm{a}, \mathrm{c}}$, R.M. Cannon ${ }^{\mathrm{b}}$, G.C. Johnson ${ }^{\mathrm{c}}$, P.A. Klein ${ }^{\mathrm{d}}$, \\ R.O. Ritchie ${ }^{\mathrm{b}, \mathrm{e}, *}$ \\ ${ }^{a}$ Sandia National Laboratory, Livermore, CA, 94550, USA \\ ${ }^{\mathrm{b}}$ Materials Sciences Division, Lawrence Berkeley National Laboratory, Berkeley, \\ CA, 94720, USA \\ ${ }^{\mathrm{c}}$ Department of Mechanical Engineering, University of California, Berkeley, CA, \\ 94720, USA \\ ${ }^{\mathrm{d}}$ Franklin Templeton Investments, San Mateo, CA, 94403, USA \\ ${ }^{\mathrm{e}}$ Department of Materials Science and Engineering, University of California, \\ Berkeley, CA, 94720, USA
}

\begin{abstract}
A micromechanical model is developed for grain bridging in monolithic ceramics. Specifically, bridge formation of a single, non-equiaxed grain spanning adjacent grains is addressed. A cohesive zone framework enables crack initiation and propagation along grain boundaries. The evolution of the bridge is investigated through a variance in both grain angle and aspect ratio. We propose that the bridging process can be partitioned into five distinct regimes of resistance: propagate, kink, arrest, stall, and bridge. Although crack propagation and kinking are well understood, crack arrest and subsequent "stall" have been largely overlooked. Resistance during the stall regime exposes large volumes of microstructure to stresses well in excess of the grain boundary strength. Bridging can occur through continued propagation or reinitiation ahead of the stalled crack tip. The driving force required to reinitiate is substantially greater than the driving force required to kink. In addition, the critical driving force to reinitiate is sensitive to grain aspect ratio but relatively insensitive to grain angle. The marked increase in crack resistance occurs prior to bridge formation and provides an interpretation for the rapidly rising resistance curves which govern the strength of many brittle materials at realistically small flaw sizes.
\end{abstract}

Key words: Fracture mechanisms, Fracture toughness, Ceramic material, Finite elements, Crack bridging

* Corresponding author.

Email address: roritchie@lbl.gov (R.O. Ritchie).

Preprint submitted to Journal of the Mechanics and Physics of Solids 9 October 2006 


\section{Introduction}

Traditionally, the use of ceramics in load-bearing structures has been severely limited by low fracture toughness. Over the last several decades, the incorporation of fracture mechanics concepts into the design of ceramics has led to marked increases in strength and toughness. In addition to increasing strength through a reduction in flaw size, there has been a focus on improving the inherent toughness of the ceramic through control of the microstructure (Evans, 1990). This approach has hinged on a thorough understanding of the driving force (Eshelby, 1951; Rice, 1968) coupled with the identification of the salient microstructural mechanisms aiding the resistance (e.g., Ritchie et al., 2000). In this regard, it is often useful to partition the mechanisms of fracture into "intrinsic" and "extrinsic" processes. Intrinsic mechanisms evolve ahead of the crack tip (independent of crack size) while extrinsic mechanisms invariably evolve behind the crack tip (and dominate resistance-curve behavior). In non-transforming ceramics, grain and/or grain boundary fracture join a host of extrinsic mechanisms which may act in the crack wake (microcracking) and across the crack surfaces (grain bridging, grain sliding) to provide resistance to crack propagation. Experimental findings indicate that for most monolithic (non-transforming) structural ceramics, the most potent extrinsic mechanism is grain bridging.

In this study, we attempt to provide a new understanding of the source of toughening during the initial stages of grain bridging. We initially review prior experimental, analytical, and computational studies on grain bridging. Although most of these studies have centered on long-crack toughness and the plateau of the resistance curve, the focus of the current work is obtaining a fundamental understanding of bridge formation and the initial rise in the resistance curve. A cohesive approach to fracture is adopted and applied to a single, non-equiaxed, inclined grain spanning adjacent grains. We propose that the grain bridging process can be broadly partitioned into five regimes of resistance. Numerical studies varying grain size, shape, and orientation support the proposed partition. Substantial toughening occurs after initial crack deflection and prior to bridge formation; moreover, for a broad range of grain angles, bridge formation occurs via crack reinitiation ahead of the primary, deflected crack tip. A rationale for bridge formation is developed for idealized microstructures and applied to structural ceramics. Crack reinitiation ahead of the primary, deflected crack tip is energetically favorable to continued propagation. We believe that the proposed toughening mechanism operable

prior to bridge formation may provide a basis for the rapidly rising resistance curves seen in many ceramics, such as silicon carbide (Gilbert et al., 1996), silicon nitride (Kruzic et al., 2005a), and dry alumina (Kruzic et al., 2005b). 


\section{Background}

Early experimental studies of Davidge and Tappin (1968) and Evans and Tappin (1972) first identified the importance of intergranular fracture in polycrystalline ceramics. Although Lange (1973) and Hübner and Jillek (1977) cited resistance curve (R-curve) behavior in elongated $\mathrm{Si}_{3} \mathrm{~N}_{4}$ and equiaxed $\mathrm{Al}_{2} \mathrm{O}_{3}$, respectively, Knehans and Steinbrech (1982) first noted that mechanisms in the crack wake enabled a rising $\mathrm{R}$-curve in $\mathrm{Al}_{2} \mathrm{O}_{3}$. Subsequent efforts by Steinbrech et al. (1983) and Swain (1986) confirmed such R-curve behavior. An exhaustive study by Swanson et al. (1987) provided indisputable, micrographic evidence of grain bridging in $\mathrm{Al}_{2} \mathrm{O}_{3}$.

Major efforts have been devoted to toughening $\mathrm{Si}_{3} \mathrm{~N}_{4}$ through the incorporation of elongated grains and weak grain boundaries (Li et al., 1992; Sajgalik et al., 1995; Van Weeren and Danforth, 1996; Becher et al., 1998). Corresponding efforts in SiC (Padture, 1994; Lee et al., 1994a,b; Cao et al., 1996; Gilbert et al., 1996; MoberlyChan and DeJonghe, 1998) focused on morphology and interfacial chemistry for $\mathrm{SiC}$.

Quantitatively, the results of this work have been impressive. Compared to an inherent fracture toughness of between 2 and $3 M P a \sqrt{m}$, toughnesses well over $10 M P a \sqrt{m}$ for $\mathrm{Si}_{3} \mathrm{~N}_{4}$ and $\sim 9 M a \sqrt{m}$ for $\mathrm{SiC}$ have been achieved. However, these values pertain to the long-crack toughness, i.e., after significant crack extension at the plateau of the R-curve.

Much of the research focused on long-crack behavior as this defines the highest toughness. Correspondingly less effort was devoted to quantifying the initial stages of the R-curve, although Xu et al. (1995) and Becher et al. (1996) did attempt to correlate microstructural parameters with short-crack toughness. It is important to note here that for structural application, the initial slope of the R-curve is in many respects more important than the peak (long-crack) toughness as it governs the strength of the ceramic at realistically small crack sizes (Kruzic et al., 2005a). Indeed, recent studies by Kruzic (Kruzic et al., 2004, 2005a,b) on $\mathrm{Al}_{2} \mathrm{O}_{3}$ and Satet et al. (2006) on $\mathrm{Si}_{3} \mathrm{~N}_{4}$ have focused on this critical issue and have shown that $\mathrm{R}$-curves can rise rapidly for crack extensions of only a few grain diameters. In addition, studies in $\mathrm{Al}_{2} \mathrm{O}_{3}$ confirm that stronger grain boundaries facilitate a rapid rise but lower plateau in the R-curve.

The methodologies employed to model grain bridging evolved with experimental findings. Early studies by Bilby et al. (1977), Lo (1978), Cotterell and Rice (1980), and Faber and Evans (1983) examined the increased toughness due to

the deflection of the crack. Additional studies quantifying the role of modulus mismatch at grain boundaries were conducted by He and Hutchinson (1989) 
and Hutchinson and Suo (1991).

With definitive observations of a bridging zone, Mai and Lawn (1987) introduced the notion of a "stress-separation" function, or in the context of the present discussion, a phenomenological cohesive zone model. This work was extended to incorporate microstructural aspects of well-aligned, weaklybonded grains through a prototypical shear-lag model (Lathabai and Lawn, 1989; Bennison and Lawn, 1989; Chantikul et al., 1990). More recently, Kovalev (Kovalev et al., 1999, 2000) proposed that miss-aligned, interlocking grains generate the majority of bridging forces and that residual clamping forces generated from thermal expansion anisotropy, the basis for the shearlag model, do not play a role.

The constitutive models governing surface separation are termed phenomenological because they stem from the evolution of a complicated (and unresolved) microstructural process involving the formation and interaction of multiple bridging grains. Rather than focus on the macroscopic bridging zone, we seek to obtain a fundamental understanding of the evolution of the initial bridge (grain debonding). Prior works by Bennison and Lawn (1989) and Chantikul et al. (1990) ignored the resistance from grain debonding based on a frictional debonding model for aligned grains (Marshall and Evans, 1988). Although Kovalev et al. (2000) examined inclined grains, the study also did not consider bridge formation and only focused on the contact of debonded grains under far-field tension. We will show that the driving forces required for bridge formation should not be ignored and that significant toughening occurs beyond simple deflection. The increased resistance beyond deflection yet prior to actual bridge formation may provide a basis for the rapidly rising resistance curves that are experimentally observed in many monolithic structural ceramics.

\section{Theory and Implementation}

A general cohesive framework employs independent constitutive models to govern bulk deformation and surface separation. The global traction distribution (along the cohesive surface) is not assumed, but results from interaction between the selected bulk and surface models. This approach was pioneered in the finite element method by Needleman and Tvergaard (Needleman, 1987, 1990; Tvergaard and Hutchinson, 1990, 1992) and numerous other contributors in the early 1990's. Initial formulations and applications of cohesive zone methods are reviewed by Klein et al. (2001).

More recently, cohesive methods have been applied to grain boundary fracture by Helms et al. (1999), where microstructure was generated via Voronoi 
tessellation and cohesive surface elements were seeded along grain boundaries. In a series of papers (Zavattieri and Espinosa, 2001; Zavattieri et al., 2001; Espinosa and Zavattieri, 2003a,b; Zavattieri and Espinosa, 2003) Zavattieri, Espinosa and coworkers examined the dynamic fragmentation of ceramic microstructures, with both digitized and synthesized microstructures being considered. Substantial efforts were taken to include grain orthotropy, grain size and shape, distributions of interface properties (fracture strength, fracture energy), and representative volume element (RVE) size. Although the framework is sufficiently general, the predicted response (microcracking, fragmentation) reflects the imposed, dynamic loading. Maiti and Geubelle (2004) developed a similar framework to capture dynamic, intergranular, branching of alumina. Although findings from the dynamic fracture of ceramic microstructures yield useful global quantities, the details of initiation and propagation of multiple interacting crack tips are difficult to discern.

This literature has demonstrated that modeling intergranular fracture in ceramic microstructures can be considered as a natural extension of the cohesive surface methodology. Rather than attempt to model digitized or synthesized microstructure, we consider the dominant extrinsic mechanism, grain bridging. Specifically, we will employ a cohesive framework to systematically investigate the role of grain size, shape, and orientation on bridge formation.

\subsection{A framework for grain bridging}

In an effort to shed light on short-crack toughness and the rapid rise in the Rcurve, we employ the finite element method to model the bridging of a single, non-equiaxed, inclined grain. Because the length scales $(\mathrm{nm})$ associated with modeling microstructure are not amenable to modeling specimen geometries $(\mathrm{cm})$, we employ a $K$-field displacement boundary condition. A schematic of the model is illustrated in Figure 1. A two-dimensional disk of radius $r$ contains a grain of length $g_{l}$, width $g_{w}$, and inclination $\theta$ spanning two adjacent grains. The origin of the $K$-field coincides with the projected crack tip position $\left(a_{p}, 0\right)$. Each crack tip $\mathbf{a}_{i}$ is "tracked" by monitoring the peak traction in the set of cohesive surface elements. Given a propagation direction $\left(\mathbf{d}=\mathbf{E}_{1}\right)$, the crack tip projection is $a_{p}=\max \left(\mathbf{a}_{i} \cdot \mathbf{d}\right)$. The "effective" measure employed for multiple crack tips differs from a direct projection of the primary crack tip $a_{p p}=\mathbf{a}_{\text {primary }} \cdot \mathbf{d}$. For a single crack, the definitions coincide $a_{p p}=a_{p}$.

As noted in Figure 1, we limit crack propagation to the grain boundaries. Grain (bulk) deformation (plane strain) is modeled through four-node, quadrilateral elements, while grain boundary (surface) behavior is modeled through fournode, line cohesive surface elements. The current framework enables finite deformations, spatially variable moduli, thermal residual stresses, and grain 


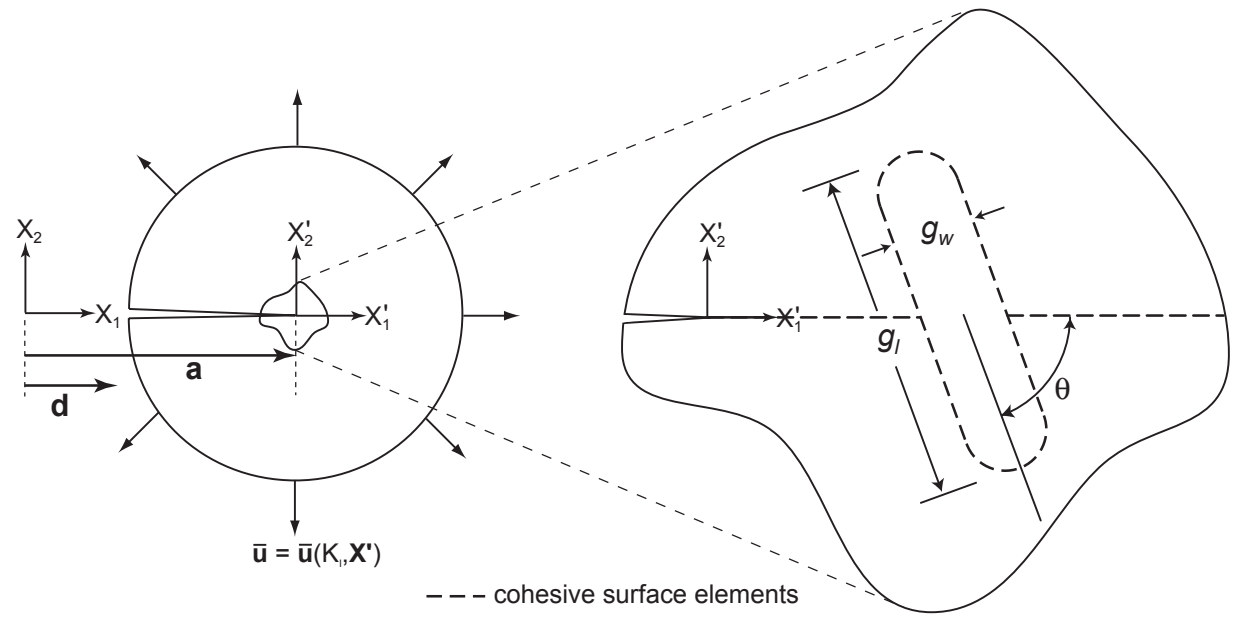

Fig. 1. An idealized two-dimensional framework for grain bridging. $K$-field boundary conditions are applied to a disk containing a non-equiaxed, inclined grain spanning two adjacent grains. Cohesive surface elements seeded along grain boundaries enable intergranular fracture.

boundary fracture (initiation, propagation, sliding). Distributions of material properties may also be incorporated for sensitivity studies.

Although the current 2-D model is idealized, we can systematically vary both material properties and "microstructure" to increase our understanding. We should note that ceramicists do not enjoy such luxuries. Processing methodologies designed to vary grain shape, size, and distribution inevitably affect grain boundary properties. The goal of the current study is to establish trends with regard to grain shape, size, and orientation. Pending the results of thorough numerical studies, we can systematically and incrementally incorporate complexity. We currently specify that grain moduli are isotropic and spatially uniform. Residual stresses and grain boundary friction are neglected. Grain boundary fracture is governed by only two parameters, grain boundary strength and grain boundary energy.

Modeling the evolution of propagation is accomplished through the concatenation of quasi-static and implicit dynamic simulations. Initial finite deformation simulations are quasi-static. After a period of stable propagation, an instability $\left.\frac{\partial J}{\partial a}\right|_{K} \geq \frac{\partial R}{\partial a}$ prohibits convergence. We emphasize $\left.\right|_{K}$ because the $K$ field boundary condition evolves with crack tip location. Consequently, during mode I propagation, the applied stress intensity is held constant $\left.\frac{\partial J}{\partial a}\right|_{K}=0$. The resistance governs stability $\frac{\partial R}{\partial a}>0$. At instability, a branch of the solution is obtained through implicit dynamics. For all simulations, dynamic arrest/fracture occurs rapidly and well before boundary reflections corrupt the solution. If the crack arrests, quasi-static simulations are subsequently restarted to further increment the far-field driving force. 


\subsection{Grain and grain boundary constitutive models}

In the current study, we partition the material response into relatively simple bulk and surface constitutive models having few material parameters. Bulk deformations are governed by a finite deformation constitutive model proposed by Simo et al. (1985), Simo and Hughes (1997). The stored energy function $\Phi$ is composed of volumetric $U$ and deviatoric $\bar{\Phi}$ parts

$$
\begin{aligned}
\Phi & =U(J)+\bar{\Phi}(\overline{\mathbf{b}}) \\
U(J) & =\frac{1}{2} \kappa\left[\frac{1}{2}\left(J^{2}-1\right)-\ln J\right] \\
\bar{\Phi}(\overline{\mathbf{b}}) & =\frac{1}{2} \mu(\operatorname{tr} \overline{\mathbf{b}}-3),
\end{aligned}
$$

where $\kappa$ is the bulk modulus, $\mu$ is the shear modulus, $\overline{\mathbf{b}}=J^{-2 / 3} \mathbf{b}$ is the isochoric part of the left Cauchy-Green stretch tensor $\mathbf{b}$, and $J=\operatorname{det} \mathbf{F}$. The resulting Cauchy stress tensor (Simo and Hughes, 1997) is

$$
\boldsymbol{\sigma}=\frac{1}{J}\left[\left(\frac{\kappa}{2}\left[J^{2}-1\right]-\frac{\mu}{3} \operatorname{tr} \overline{\mathbf{b}}\right) \mathbf{I}+\mu \overline{\mathbf{b}}\right] .
$$

We note that for small deformations, the bulk model reduces to isotropic, linear elasticity. This generalization enables a consistent application of the $K$-field boundary condition and a proper treatment of the large, local deformations (strains and rotations) that accompany bridge formation.

Cohesive surface relations are taken from Xu and Needleman (1994). For this work, we postulate that the normal fracture energy $\phi_{n}$ and the shear fracture energy $\phi_{t}$ are equivalent. The resulting components of the traction are

$$
T_{n}(\boldsymbol{\Delta})=\phi_{n} \frac{\Delta_{n}}{\delta_{n}^{2}} \exp \left(-\frac{\Delta_{n}}{\delta_{n}}\right) \exp \left(-\frac{\Delta_{t}^{2}}{\delta_{t}^{2}}\right)
$$

and

$$
T_{t}(\boldsymbol{\Delta})=2 \phi_{n} \frac{\Delta_{t}}{\delta_{t}^{2}}\left(1+\frac{\Delta_{n}}{\delta_{n}}\right) \exp \left(-\frac{\Delta_{n}}{\delta_{n}}\right) \exp \left(-\frac{\Delta_{t}^{2}}{\delta_{t}^{2}}\right) .
$$

where $\delta_{n}$ and $\delta_{t}$ are the characteristic length scales governing normal and tangential separation, respectively. The normal and tangential openings are defined as $\Delta_{n}=\boldsymbol{\Delta} \cdot \mathbf{n}$ and $\Delta_{t}=\boldsymbol{\Delta} \cdot \mathbf{t}$ with respect to the local normal $\mathbf{n}$ and tangent $\mathbf{t}$ to the cohesive surface element.

Because the form of the separation law is specified, one can find the normal strength $\sigma_{\max }$ and shear strength $\tau_{\max }$ through the fracture energies and characteristic lengths as

$$
\sigma_{\max }=\frac{\phi_{n}}{e \delta_{n}} \quad \tau_{\max }=\sqrt{\frac{2}{e}} \frac{\phi_{t}}{\delta_{t}}
$$


where $e=\exp [1]$. Frequently, one assumes that the characteristic lengths are equivalent, $\delta_{n}=\delta_{t}$. Given $\phi_{t}=\phi_{n}$, we find that the shear strength exceeds the normal strength, $\tau_{\max }=\sqrt{2 e} \sigma_{\max } \sim 2.3 \sigma_{\max }$. For the majority of solids, the theoretical tensile strength exceeds the theoretical shear strength (Macmillan, 1972). We postulate that this observation can be extended to bound the grain boundary shear strength $\tau_{\max }=\sigma_{\max }$ and find $\delta_{t}=\sqrt{2 e} \delta_{n}$. The resulting constitutive models for normal and shear separation are displayed in Figure 2.

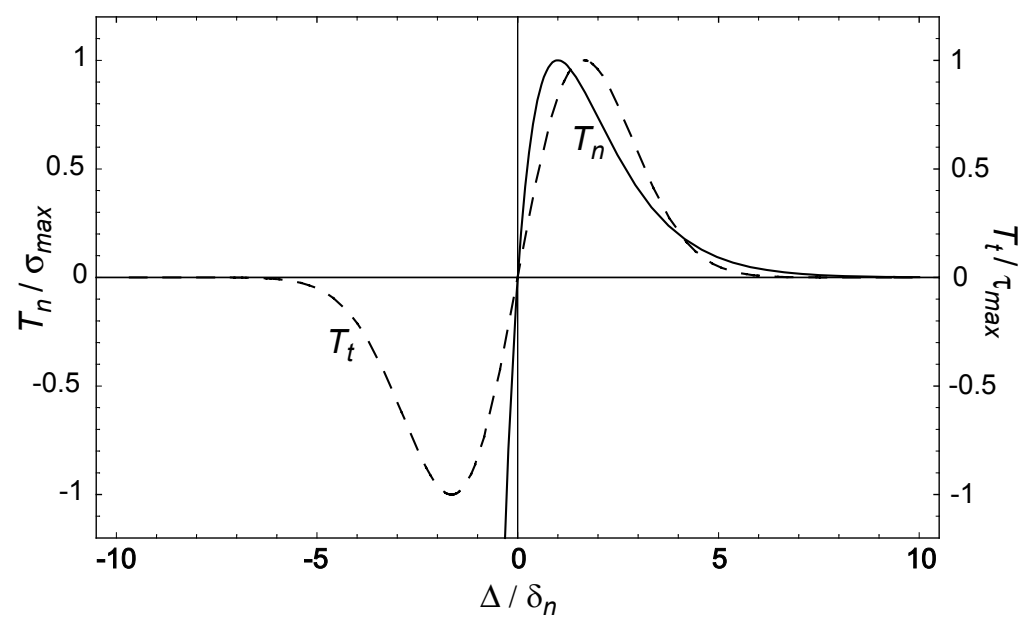

Fig. 2. Normalized constitutive model (Xu and Needleman, 1994) for surface separation. The shear boundary strength coincides with the normal boundary strength, $\delta_{t}=\sqrt{2 e} \delta_{n} \rightarrow \tau_{\max }=\sigma_{\max }$. The fracture energy is independent of the mode of loading, $\phi_{t}=\phi_{n}$.

Care must be taken when employing the current surface model for mixedmode loadings. For $\phi_{t}=\phi_{n}$, the shear traction contains a normal multiplier $N\left(\Delta_{n}\right)=\left[1+\frac{\Delta_{n}}{\delta_{n}}\right] \exp \left(-\frac{\Delta_{n}}{\delta_{n}}\right)$. Surface interpenetration will affect both the shear strength and fracture energy. As $\Delta_{n} \rightarrow-\delta_{n}, N \rightarrow 0$. Consequently, contact surfaces are employed to minimize interpenetration. One must also address unloading; because we cannot exclude grain boundary healing, we do not stipulate an additional model for unloading. We do, however, address complete boundary separation through a gap cutoff. If $\Delta_{n}>7 \delta_{n}$ or $\Delta_{t}>7 \delta_{t}$, we transition to a free surface. For normal separation, $T_{n}=0.017 \sigma_{\max }$ and $\varphi=0.99 \phi_{n}$ at $\Delta_{n}=7 \delta_{n}$. For shear separation, $T_{t}=0.001 \tau_{\max }$ and $\varphi=1.00 \phi_{n}$ at $\Delta_{t}=7 \delta_{t}$.

In this work, cohesive surface elements are only employed along the grain boundaries. Consequently, the additional compliance (softening) introduced by the cohesive surface model is negligible. Softening for a cohesive surface network is addressed in Klein et al. (2001). The effective moduli of the composite material mirrors the moduli of the bulk material. 


\subsection{Material properties}

Because the study targets mechanisms, grain and grain boundary properties are not aligned with a specific material system but are chosen to be representative of monolithic ceramics. The grain bulk modulus $\kappa$ and shear modulus $\mu$ are derived from macroscopic measurements of Young's modulus $E$ and Poisson's ratio $\nu$. For this study, we only specify the normal grain boundary fracture energy $\phi_{n}$ and the normal grain boundary strength $\sigma_{\max }$. We assume that the normal fracture energy $\phi_{n}$ coincides with the mode I critical driving force $J_{0}$. The critical driving force can be correlated with the threshold of the $R$-curve while the normal grain boundary strength is assumed to be a fraction of Young's modulus E/30. Material parameters for $E$, $\nu$, and $J_{0}$ were taken from the work of Vekinis et al. (1990) on $\mathrm{Al}_{2} \mathrm{O}_{3}$. Although the moduli are more representative of $\mathrm{Si}_{3} \mathrm{~N}_{4}$ and the experiments focus on long-crack toughness, the parameters reflect monolithic ceramics. Given $J_{0}$ and $\sigma_{\text {max }}$, the grain boundary shear strength $\tau_{\max }$ and characteristic length scales $\delta_{n}, \delta_{t}$ can be deduced. Properties for the model system are presented in Table 1.

Table 1

Grain and grain boundary properties of the model, ceramic system.

\begin{tabular}{|c|c|c|c|}
\hline Microstructure & Property & Ceramic & Units \\
\hline \multirow{4}{*}{ Grain } & $\kappa$ & 174 & $G P a$ \\
\cline { 2 - 4 } & $\mu$ & 125 & $G P a$ \\
\cline { 2 - 4 } & $E$ & 303 & $G P a$ \\
\cline { 2 - 4 } & $\nu$ & 0.21 & - \\
\cline { 2 - 4 } & $\rho$ & 3.96 & $\mathrm{~kg} / \mathrm{m}^{3}$ \\
\hline \multirow{5}{*}{ Grain boundary } & $J_{0}$ & 40. & $\mathrm{~J} / \mathrm{m}^{2}$ \\
\cline { 2 - 4 } & $K_{0}$ & 3.6 & $\mathrm{MPa} \sqrt{m}$ \\
\cline { 2 - 4 } & $\phi_{n}$ & 40. & $\mathrm{~J} / \mathrm{m}^{2}$ \\
\cline { 2 - 4 } & $\phi_{t}$ & 40. & $\mathrm{~J} / \mathrm{m}^{2}$ \\
\cline { 2 - 4 } & $\sigma_{\max }$ & 10. & $G P a$ \\
\cline { 2 - 4 } & $\tau_{\max }$ & 10. & $G P a$ \\
\cline { 2 - 4 } & $\delta_{n}$ & 1.5 & $\mathrm{~nm}$ \\
\cline { 2 - 4 } & $\delta_{t}$ & 3.4 & $\mathrm{~nm}$ \\
\hline
\end{tabular}

A close examination of Table 1 reveals the challenge of employing cohesive surface models in the study of ceramics. Although we have explicitly incorporated a length scale $\left(\delta_{n}, \delta_{t}\right)$ to regularize propagation, we must now adequately 
resolve the resulting cohesive zone (Klein et al., 2001). Simulations indicate that a large boundary strength coupled with a small fracture energy requires substantial mesh refinement. In fact, the issue may be further complicated by the tangent of the traction-displacement law.

\subsection{Numerical study}

Prior to investigating sensitivity to grain and grain boundary properties, we seek to obtain a greater understanding of the grain bridging process through a variation of grain size, shape, and angle. We propose to fix the grain width $g_{w}$ and vary the grain length $g_{l}$ and grain angle $\theta$. For a grain width of $150 \mathrm{~nm}$, grain lengths of 300,450 , and $600 \mathrm{~nm}$ are selected. To simplify the presentation

of our findings, grain size and shape are expressed in terms of grain aspect ratio $A R=g_{l} / g_{w}$. The resulting aspect ratios are 2, 3, and 4. Grain angles $\operatorname{span} 45^{\circ} \rightarrow 90^{\circ}$.

Three-point bend simulations employing the material properties noted in Table 1 were conducted to determine the mesh size required for mode I propagation. Cohesive surface elements sizes under $10 \mathrm{~nm}$ were sufficient to ensure both accuracy and stability. All meshes used for this work have a cohesive surface element size $h$ of $2.5 \mathrm{~nm}$.

A typical finite element mesh $\left(A R=4, \theta=60^{\circ}\right)$ is displayed in Figure 3 . The mesh is regular near the grain boundary and coarsens away from the boundary. The disk radius $r$ is $50 \mu \mathrm{m}$ and the resulting ratio of cohesive surface element size to disk radius is $1: 20,000$. The disk radius is large compared to the grain length $\left(r / g_{l}>50\right)$ and provides ample time for crack arrest or propagation before boundary reflections corrupt the dynamic solution.

Through a variation in grain aspect ratio and grain angle, this work seeks to

(1) Partition the evolution of grain bridging.

(2) Establish mechanism(s) aiding initial R-curve behavior prior to bridge formation.

\section{Findings}

We seek to elucidate the grain bridging process by partitioning the evolution into several regimes. The applied driving force $J$, derived from the applied, mode I, $K$-field boundary condition, is normalized by the critical driving force for the grain boundary fracture $J_{0}$ to obtain a metric for bridging $J / J_{0}$. Because the grain boundary fracture energy is equivalent for all modes of loading, 


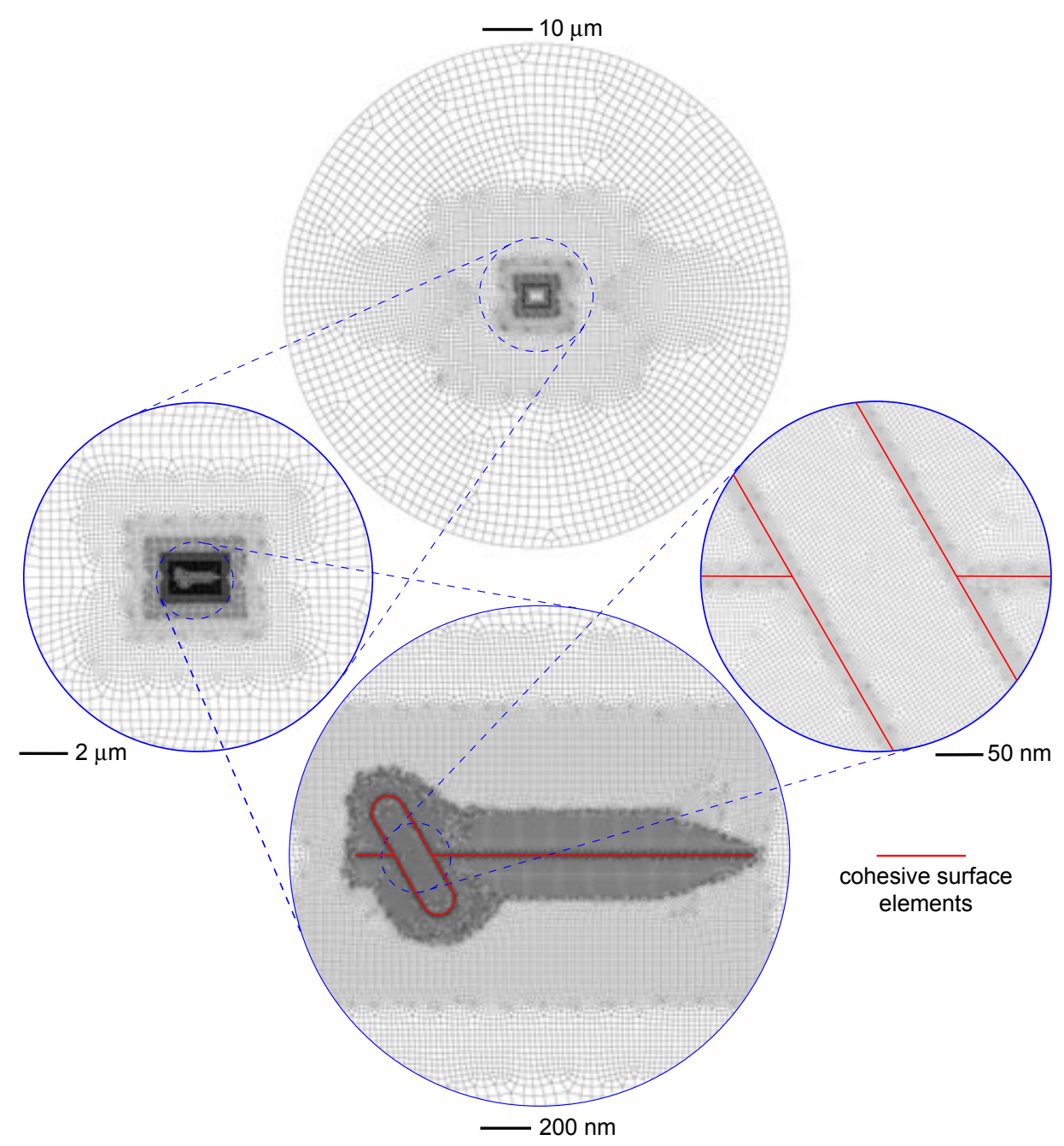

Fig. 3. Typical mesh used in the analysis of grain bridging. Cohesive surface elements are seeded along refined grain boundaries. The radius of the disk $r$ is $50 \mu \mathrm{m}$ and the cohesive surface element size $h$ is $2.5 \mathrm{~nm}$.

$J_{0}=\phi_{n}=\phi_{t}, J / J_{0}$ represents the ratio of far-field loading to grain boundary resistance.

Each simulation at a particular aspect ratio and grain angle can be viewed as series of events which are defined by the driving force required to propagate $J^{p}$, kink $J^{k}$, arrest $J^{a}$, stall $J^{s}$, and bridge $J^{b}$. Figure 4 schematically illustrates the partition of the grain bridging process. Coordinates $\left(a_{p p}, J / J_{0}\right)$ of the proposed partition are displayed in Figure 4 . We note that the crack tip position given in Figure 4, $a_{p p}$, represents a direct projection of the primary crack tip.

Following Figure 4, the crack propagates at constant driving force $J=J_{0}$ until it intersects the grain boundary of the single, inclined grain. Two crack tips form along the bridging grain boundary. The far-field load is increased until 
the $\operatorname{crack}(\mathrm{s})$ kinks at $J^{k}$ along the flank(s) of the inclined grain. Additional driving force is required to propagate the post-kink crack along the grain flank. Unstable propagation ensues and the crack arrests on the far side of the inclined grain at $J=J^{a}$. Continued loading yields incremental crack growth. The primary crack is essentially "stalled." Further loading results in another instability at $J^{s}$. Unstable propagation along (and up) the far grain flank or reinitiation ahead of the primary crack tip results in bridge formation at $J^{b}$.

Figure 5 illustrates the near-tip stresses at each regime transition for two cases with grain aspect ratio $A R=3$ and grain angles $\theta=90^{\circ}, 45^{\circ}$. Both cases in Figure 5 exhibit reinitiation ahead of the primary crack tip. For ease, we refer to continued propagation along and up the far grain flank as crack climbing. Because the proposed partition holds for almost every case, we report and discuss our findings in the context of $J^{p} / J_{0}, J^{k} / J_{0}, J^{a} / J_{0}, J^{s} / J_{0}$, and $J^{b} / J_{0}$. Each loading regime in the bridging process is spanned by these normalized driving forces. We now proceed to discuss each regime in more detail.

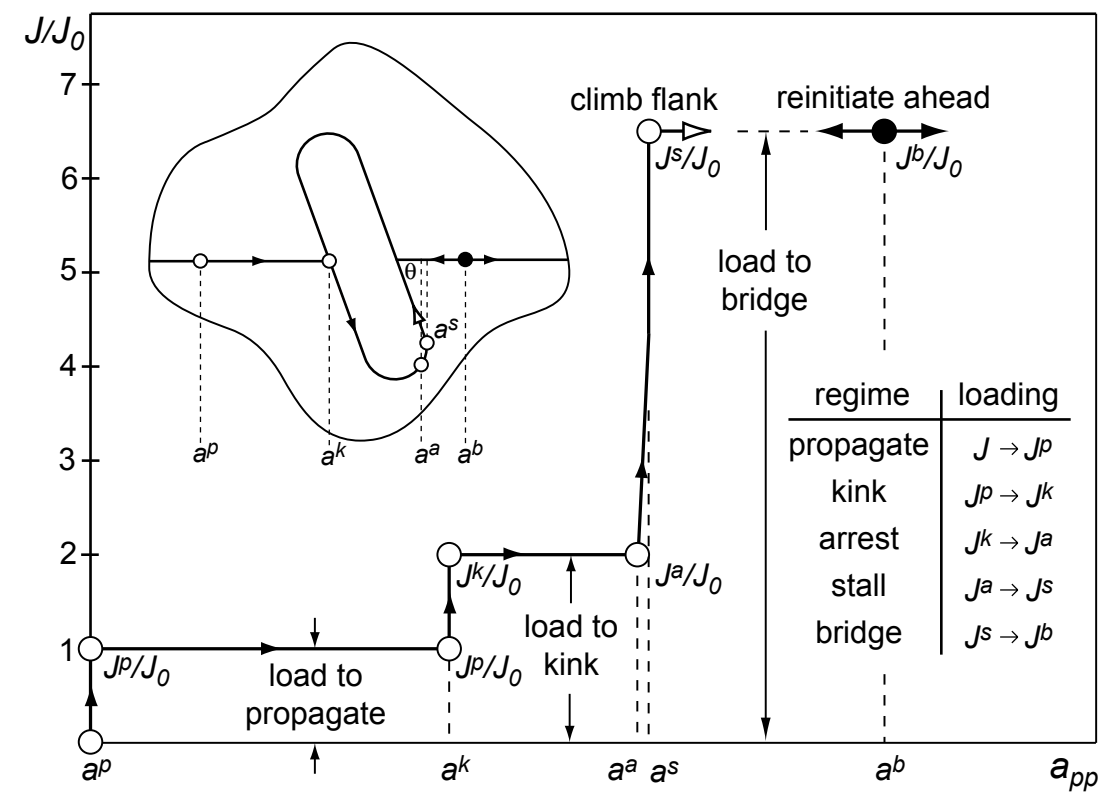

Fig. 4. Partition of the grain bridging process into propagate, kink, arrest, stall, and bridge. The normalized driving force $J / J_{0}$ is plotted against a direct projection of the primary crack tip $a_{p p}$. The driving force required to bridge $J^{b}$ is substantially greater than the driving force required to kink $J^{k}$.

\section{$4.1 \quad$ Propagate $J \rightarrow J^{p}, a^{p} \rightarrow a^{k}$}

The first regime is defined by the driving force required to propagate the crack along the grain boundary in mode I. A cohesive zone forms at the pre-crack and the crack propagates when $J=J^{p}$. Because the mode of loading $\left(J \mathbf{E}_{1}\right)$ 

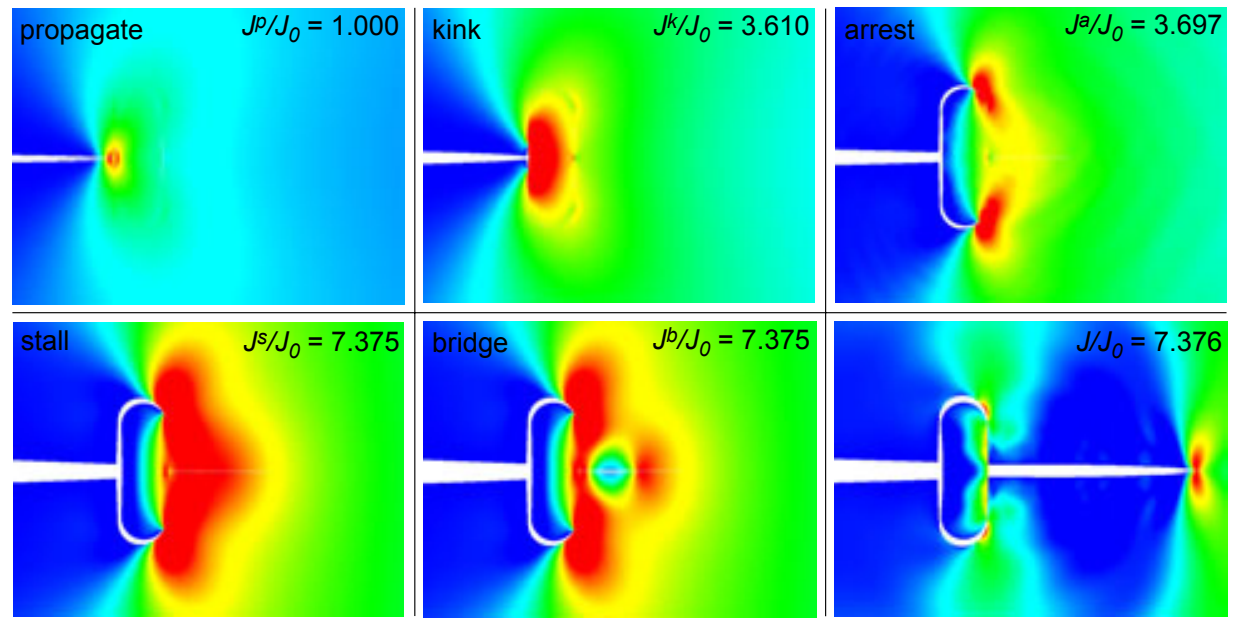

(a)
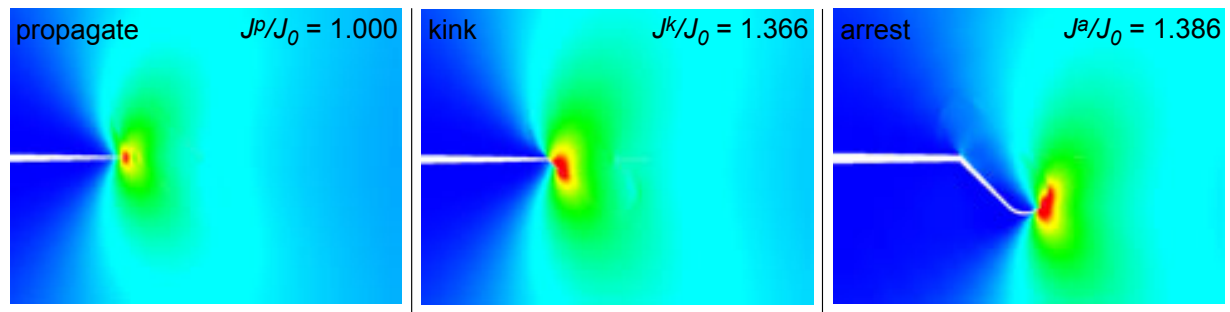

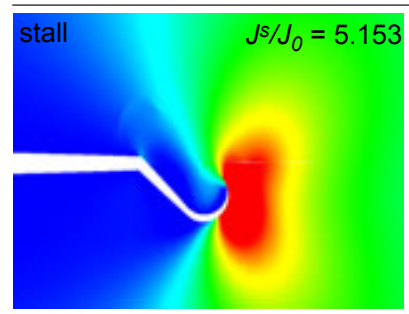

$300 \mathrm{~nm}$

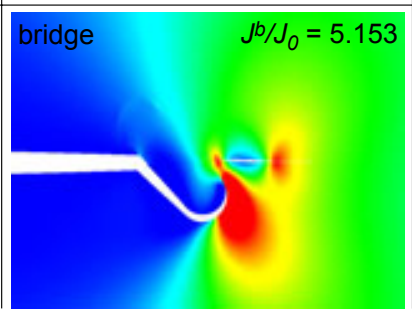

(b)

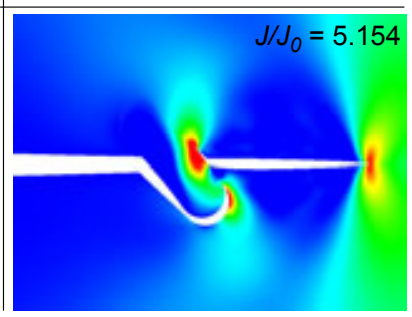

$\sigma_{22}(\mathrm{GPa})$

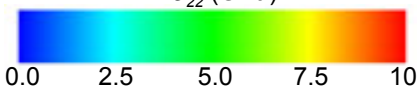

Fig. 5. Evolution of grain bridging for $A R=3$. Each step of (a) $90^{\circ}$ and (b) $45^{\circ}$ corresponds to a normalized driving force $J / J_{0}$ noted in Figure 4.

is parallel to the pre-crack configuration $\left(a \mathbf{E}_{1}\right), J^{p}=J_{0}$ for all simulations. Figure 5 illustrates the extent of the highly stressed volume $\left(\sigma_{22}>10 \mathrm{GPa}\right)$ at $J^{p} / J_{0}=1$ for two representative simulations. As the crack propagates, the $K$-field boundary conditions are updated to reflect the current crack tip location and provide a constant driving force.

During propagation, the cohesive zone size $l_{p z}$ is approximately $50 \mathrm{~nm}$. The cohesive zone size is defined as the distance from the peak $T_{n}=\sigma_{\max }$ to the tail $T_{n} \sim 0.1 \sigma_{\max }$ of the global traction distribution. We note that $0.1 \sigma_{\max }$ corresponds to the majority of the fracture energy, $0.96 \phi_{n}$. A cohesive surface element size $h$ of $2.5 \mathrm{~nm}$ ensures that the fields are well resolved and that the system is stable. 


\subsection{Kink $J^{p} \rightarrow J^{k}, a^{k}$}

The second regime is defined by the driving force required to kink the crack. The crack propagates at $J_{p} / J_{0}=1$ until the tip nears the inclined, bridging grain. A slight increase in $J\left(\sim 1.05 J^{p} / J_{0}\right)$ is sufficient to drive the crack into the intersecting grain boundary. Subsequent loading forms two cohesive zones along the flanks of the bridging grain. When $J=J^{k}$, sufficient driving force exists to propagate or "kink" the $\operatorname{crack}(\mathrm{s})$ along the flank(s) of the grain. Although we have adopted the term kink from the literature, the process is smooth. Figure 5 illustrates the marked difference in the resistance at the extremes of grain angle. The normalized driving force to kink at $\theta=90^{\circ}$ is over two and a half times the normalized driving force to kink at $\theta=45^{\circ}$.

To enable a clear comparison between simulated and analytical findings, another series of simulations was conducted to model a single kink. For these simulations, cohesive surface elements were not seeded along the upper grain flank and the crack was constrained to deflect down along the lower grain flank. The comparison between simulated and analytical (He and Hutchinson, 1989) findings is illustrated in Figure 6. As indicated in Figure 6, the simulated kinking condition does not depend on grain length $g_{l}$ and the resulting aspect ratio $A R$. Differences between simulated and analytical findings are minimal but exist for all grain angles.

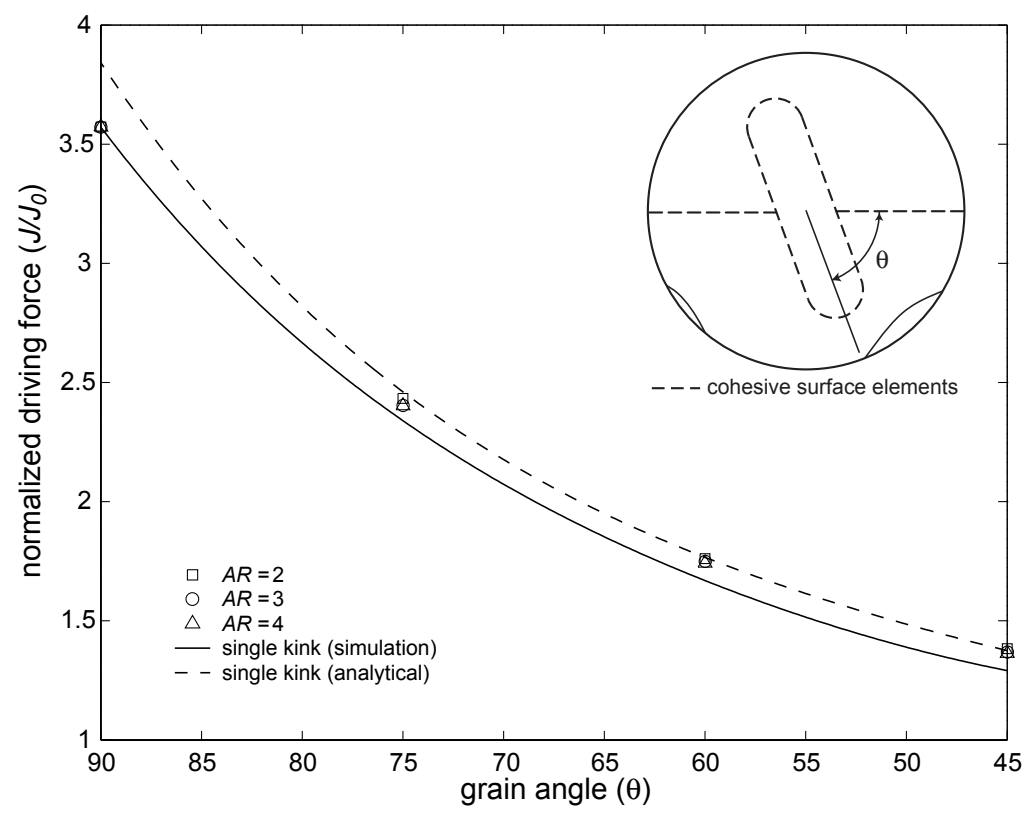

Fig. 6. Comparison between simulated and analytical (He and Hutchinson, 1989) findings for the kink condition, $J / J_{0}=J^{k} / J_{0}$. The kink condition is independent of aspect ratio $A R$. 
Further studies at a particular grain angle $\left(\theta=60^{\circ}\right)$ varied the cohesive zone size $l_{p z}$ through changes in cohesive strength $\sigma_{\max }(E / 60 \rightarrow E / 10)$, keeping the fracture energy $\phi_{n}$ constant. Although $l_{p z}$ varies by an order of magnitude (10 $n m \rightarrow 100 \mathrm{~nm}$ ), the normalized load required to kink $J^{k} / J_{0}$ remains constant. Additional cases employing the grain boundary strength and fracture energy noted in Table 1 focused on grain angles larger than $90^{\circ}$. Findings for grain angles $135^{\circ} \rightarrow 45^{\circ}$ are displayed in Figure 7 . Simulated and analytical findings begin to diverge substantially for $\theta>80^{\circ}$.

We also note that principal stresses in the bridging grain (adjacent to the intersecting grain boundaries) peak prior to kinking. For $\theta=90^{\circ}$, local principal stresses reach $\sim E / 7$ at $J / J_{0}=2.34$. The load to kink at $\theta=90^{\circ}$ is $J^{k} / J_{0}=3.61$.

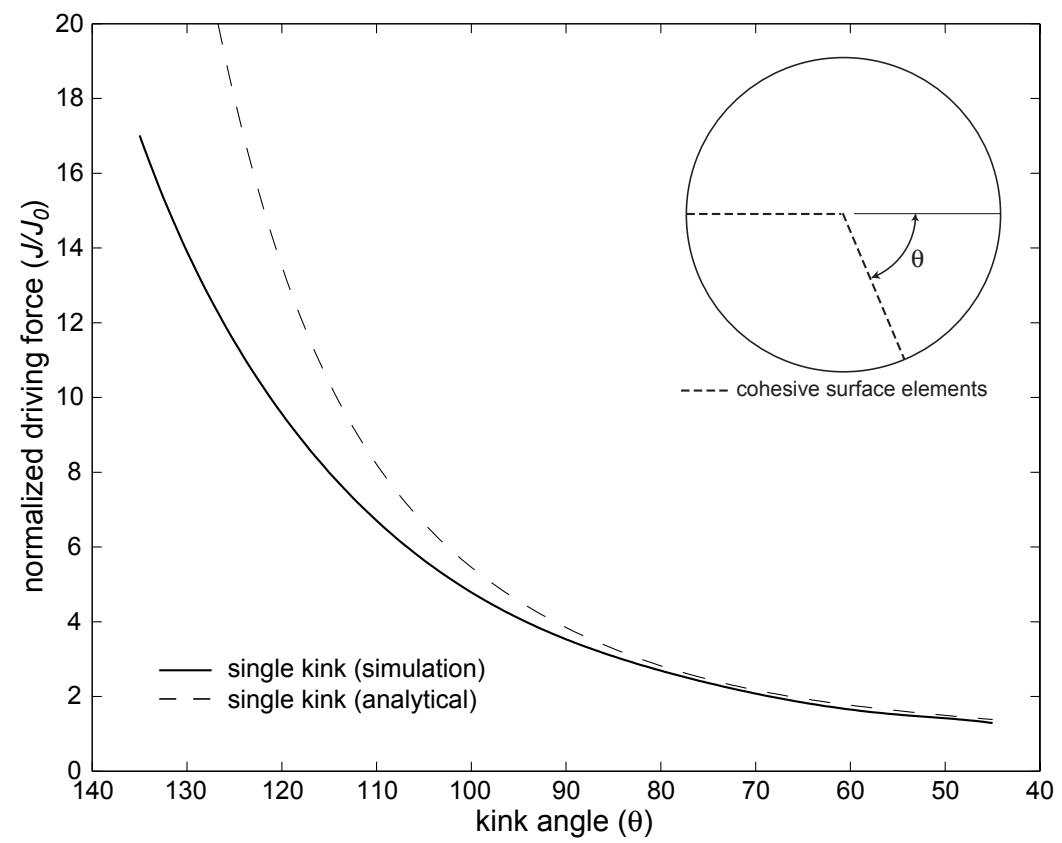

Fig. 7. Comparison between simulated and analytical (He and Hutchinson, 1989) findings for the load required to propagate a single kink $J^{k}$. Analytical findings diverge for larger grain angles.

\section{$4.3 \quad$ Arrest $J^{k} \rightarrow J^{a}, a^{k} \rightarrow a^{a}$}

The third regime is characterized by the minimal increase in driving force required to propagate and arrest the post-kink crack tip. After the crack deflects (kinks), additional driving force is required to propagate the kinked crack along flank $\left(45^{\circ}, 60^{\circ}, 75^{\circ}\right)$ or flanks $\left(90^{\circ}\right)$ of the grain. Generally, larger loading increments are required for longer, perpendicular grains. In all cases, 
a global instability $\left.\frac{\partial J}{\partial a}\right|_{K} \geq \frac{\partial R}{\partial a}$ occurs during propagation along the flank. For $A R=4, \theta=90^{\circ}$, the instability occurs at $1.03 J^{k} / J_{0}$. For the two cases displayed in Figure 5, the instability occurs at $1.02 \mathrm{~J}^{k} / J_{0}$ and $1.01 \mathrm{~J}^{k} / J_{0}$ for $A R=3, \theta=90^{\circ}$, and $A R=3, \theta=45^{\circ}$, respectively.

To ensure that the last converged quasi-static solution borders the global instability, the load step is sequentially "cut" by more than a factor of 1000 . Each simulation is restarted with implicit dynamics (trapezoidal rule with a time step $\Delta t$ of $0.1 \mathrm{~ns}$ ) to obtain a branch of the solution. An increment in the applied $K$-field, $\Delta K=0.01 K_{0}$, is applied over $10 \mathrm{~ns}(0.0036 M P a \sqrt{m} / n s)$. After the arrival of the dilatational wave from the $K$-field boundary $(5.3 \mathrm{~ns})$, the crack propagates $(\sim 5.5 \mathrm{~ns})$ and arrests in short order $(\sim 6.2 \mathrm{~ns})$. The remainder of the simulation confirms the arrest condition. Additional implicit $(\Delta t=10 \mathrm{ps})$ and explicit $(\Delta t=0.1 \mathrm{ps})$ simulations employing refined time steps confirm prior findings.

If we neglect the time (load) required for wave propagation from the boundary, the effective increment for unstable crack propagation along the near flank and arrest at the far flank is negligible $\left(<0.001 K_{0}\right)$. The applied driving force that characterizes this regime $J^{a}$ stems from the prior, small increment needed to propagate the post-kink crack tip to instability. Because the increment is $\leq 0.03 J^{k} / J_{0}$ for all cases, the schematic, Figure 4, depicts the region as essentially flat. Figure 5 illustrates differences in the kinked $J^{k} / J_{0}$ and arrested $J^{a} / J_{0}$ configurations. The only exceptions occur for $A R=2$ with $\theta=87.5^{\circ}$, $90^{\circ}$ where the crack does not arrest after instability.

\section{$4.4 \quad$ Stall $J^{a} \rightarrow J^{s}, a^{a} \rightarrow a^{s}$}

The fourth regime is characterized by the relatively large loading required to incrementally propagate the primary crack tip(s). Arrested simulations are restarted employing quasi-statics. The load step for next stage of the analysis is $0.025 K_{0}$, roughly 25 times the effective $\Delta K$ applied during the entire, previous dynamic analysis. Because the crack did not propagate during the first load step (for all cases), we confirm that the prior crack configuration at $J^{a}$ is stable.

Increasing $J$ drives the crack to the far flank of the inclined grain. Further loading does not result in continued propagation. The crack is essentially "stalled" under mixed-mode loading (see Figure 4 and Figure 5). As $J \rightarrow J^{s}$, large volumes of microstructure are exposed to stresses well in excess of the grain boundary strength $E / 30$. Depending on the grain geometry, local regions adjacent to the stalled crack tip are subjected to principal stresses between $E / 12$ and $E / 6$. The rapid rise in the resistance is expressed in Figure 4 while the 
extent of the stressed volume $\left(\sigma_{22}>\sigma_{\max }\right)$ is depicted in Figure 5. The end of the stall regime $J^{s}$ coincides with $\left.\frac{\partial J}{\partial a}\right|_{K} \geq \frac{\partial R}{\partial a}$, a second global instability.

\subsection{Bridge $J^{s} \rightarrow J^{b}, a^{s} \rightarrow a^{b}$}

The bridging regime is defined as the manifestation of propagate, kink, arrest, and stall. To understand the nature of bridge formation, quasi-static simulations were again restarted employing implicit dynamics (trapezoidal rule, $\Delta t=0.1 \mathrm{~ns}$ ). For most cases, a crack reinitiates ahead of the primary crack tip. We note that like other features of the simulation (kink, arrest, stall), the phenomena termed reinitiation is not enforced through a criterion but is a natural outcome of the simulation. Figure 8 illustrates the location of the stalled crack tip (o) and the origin of crack initiation ( + ) for $A R=3,4$ with $\theta=60^{\circ}$. All cases are displayed in Figure 9. Additional implicit $(\Delta t=10 \mathrm{ps})$ and explicit $(\Delta t=0.1 \mathrm{ps})$ simulations at various grain angles validated prior findings $(\Delta t=0.1 \mathrm{~ns})$.
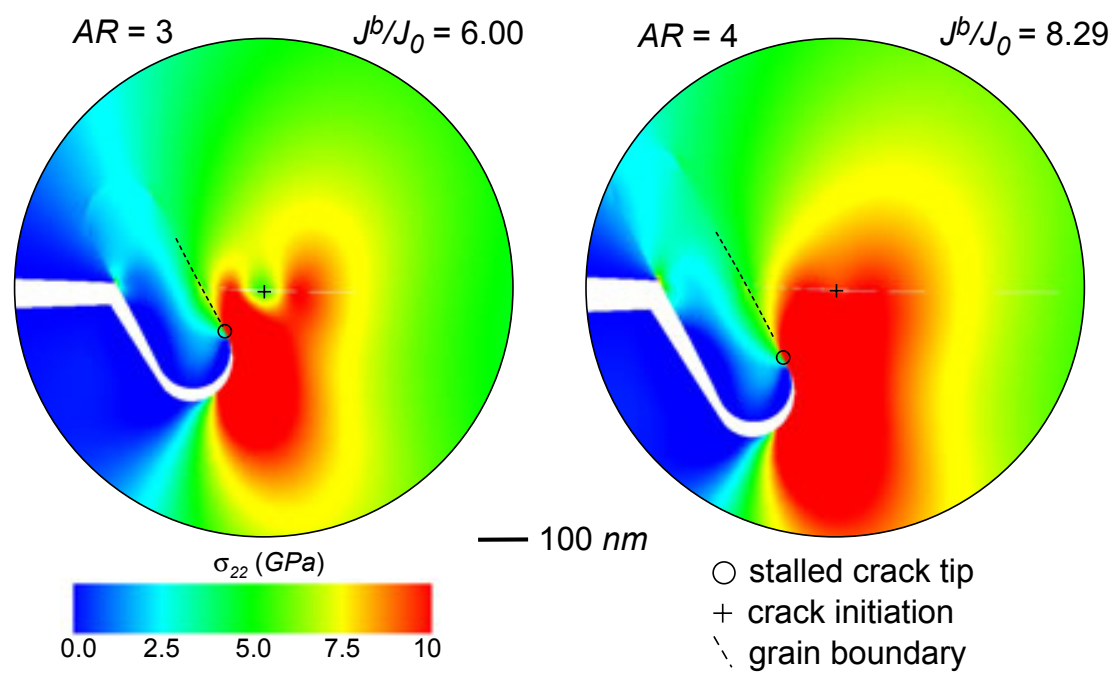

Fig. 8. Normalized peak toughness $J^{b} / J_{0}$ for $A R=3,4$ and $\theta=60^{\circ}$. The far flank of the grain is outlined to highlight reinitiation ahead of the primary crack tip. Note the volume of microstructure exposed to stresses in excess of the grain boundary strength, $\sigma_{22}>\sigma_{\max }, 10 \mathrm{GPa}$.

Although all cases for $A R=3,4$ illustrated in Figure 9 reinitiate ahead of the primary crack tip, significant crack climbing along the far grain flank occurs at $\theta=15^{\circ}$ prior to reinitiation. Consequently, the scope of the numerical study was extended to investigate deviating behavior for grain angles $90^{\circ}<\theta \leq 75^{\circ}$. Dynamics reveals that the preferred path is continued propagation along and up the far grain flank rather than reinitiation ahead of the primary crack. 


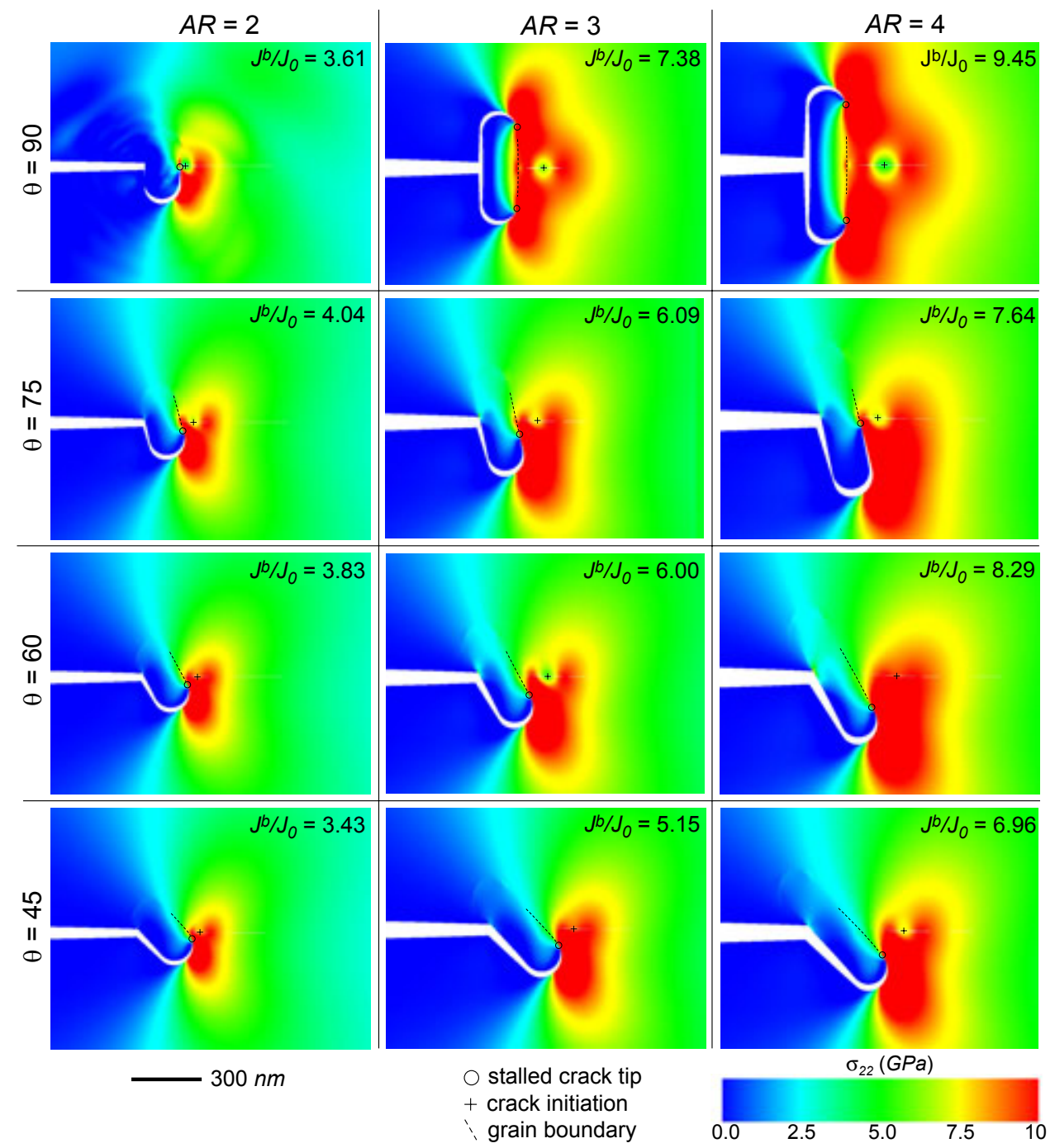

Fig. 9. Normalized peak toughness $J^{b} / J_{0}$ for the study. The far flank of the grain is outlined to highlight reinitiation ahead of the primary crack tip. Note the volume of microstructure exposed to stresses in excess of the grain boundary strength, $\sigma_{22}>\sigma_{\max }, 10 \mathrm{GPa}$.

Because the preference to climb along the far grain flank is sensitive to grain angle, numerous cases are simulated at various aspect ratios. The normalized load required to bridge $J^{b} / J_{0}$ for all simulations is illustrated in Figure 10. The normalized load required to kink $J^{k} / J_{0}$ is plotted for comparison. Because crack propagation (climb, reinitiate) nearly corresponds to arrival of the dilatational wave, $J^{b}$ is equivalent to $J^{s}$. This is reflected in the schematic of the process (Figure 4).

Re-examination of prior, quasi-static simulations of the stall regime, reveals that cohesive surface elements begin to unload $\left(\Delta_{n}>\delta_{n}\right)$ at the point of 
reinitiation. The characteristic "dip" in the cohesive surface global traction distribution is illustrated in Figure 11. Cases in which unloading does not occur ahead of the stalled crack tip correspond to a preference to climb the flank.

After reinitiation ahead of the stalled crack tip at $J^{b}$, two cracks propagate fore and aft along the initiated grain boundary. The crack propagating aft intersects the grain and forms two cohesive zones along the flanks of the grain. Prior to propagation along the flank, the mode-mixity at the stalled crack tip becomes predominantly mode II. The remaining ligament fails under shear. If the grain is not oriented at $\theta=90^{\circ}$, a bridge is formed. The bridged configuration or lack thereof is illustrated in Figure 5.

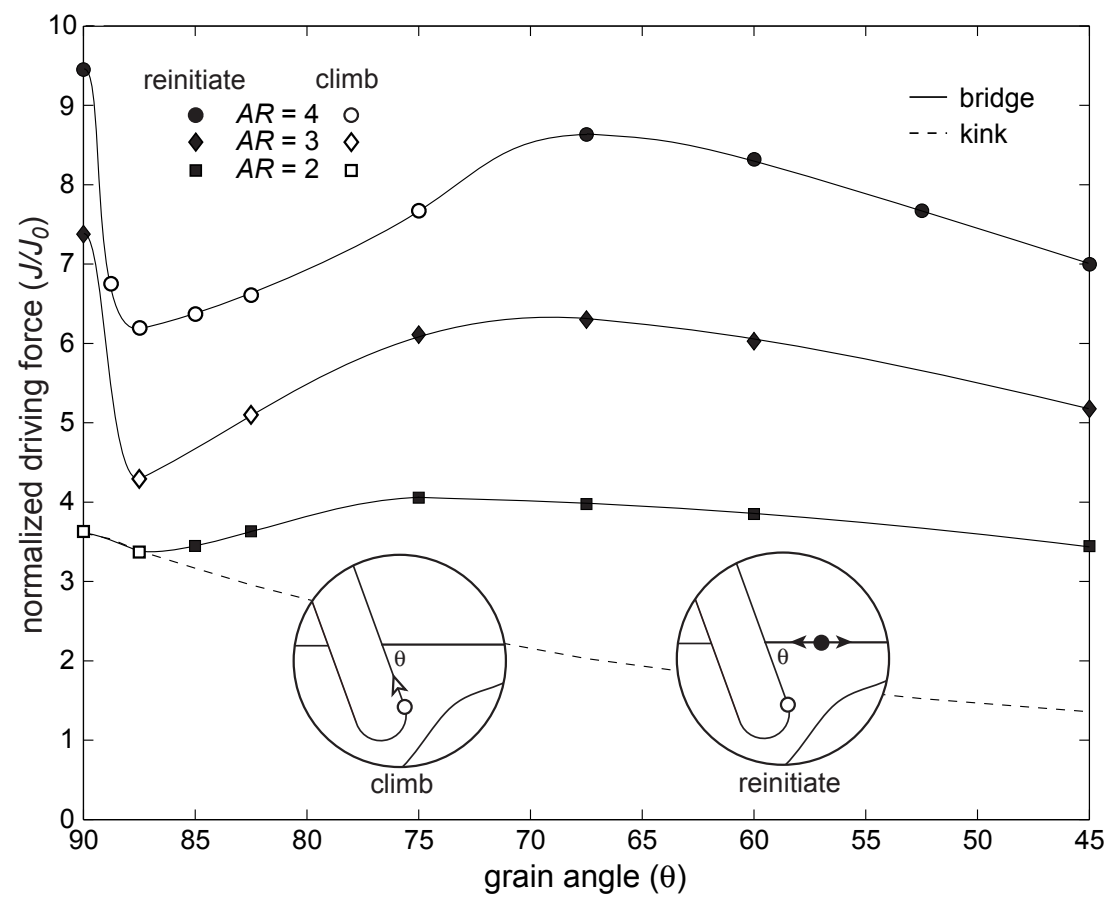

Fig. 10. Contrast of the normalized driving force required to kink $J^{k} / J_{0}$ and bridge $J^{b} / J_{0}$ for all grain angles and grain sizes. Filled symbols indicate crack reinitiation ahead of the primary crack while unfilled symbols indicate climb along the grain flank. The load required to bridge is significantly greater than the load required to kink.

\section{Discussion}

In this study, we have described a mechanistic framework for grain bridging, as applied to microstructures with varying grain aspect ratio and grain angle, and identified a potent source of toughening associated with the initial stages 
of the process. Modeling high-strength, low-toughness materials can be difficult because the cohesive zone sizes are inherently small and substantial levels of discretization are required for resolution. Numerical studies are further complicated by a fracture process that is inherently unstable. The far-field load $J$ increases monotonically; consequently, not only must the resistance $R$ in-

crease, stability requires $\frac{\partial R}{\partial a}>\left.\frac{\partial J}{\partial a}\right|_{K}$. The inequality is not always satisfied and unstable propagation occurs after the kink and stall regimes. Branches of the solution are obtained through implicit dynamics. The resulting concatenation of quasi-static and implicit dynamic simulations yields the evolution of grain bridging through multiple interacting crack tips.

\subsection{Current Findings}

Our results indicate that the evolution of crack bridging for the parameterized microstructures can be partitioned into the distinct stages of propagation, kink, arrest, stall, and bridge. Although the kink condition is well documented, differences remain between analytical and simulated findings. Those differences are magnified for kinking angles greater than $90^{\circ}$. Through a variation in the grain boundary strength $\left(E / 60<\sigma_{\max }<E / 10\right)$ and the resulting variation in the cohesive zone size $\left(10 \mathrm{~nm}<l_{p z}<100 \mathrm{~nm}\right)$, we confirm that the simulated kinking condition $J^{k}$ is independent of cohesive zone size. Kinking mirrors propagation in that if the bulk is elastic, both propagation (Willis, 1967; Rice, 1968) and kinking are only governed by the grain boundary fracture energy $\phi_{n}$.

We note that post-kink propagation is unstable and that cracks arrest in a stalled configuration. Although previous studies (Bennison and Lawn, 1989; Kovalev et al., 2000) acknowledge crack deflection and subsequent debonding prior to bridge formation, most conclude that these contributions to the toughness are negligible. The current study finds this to be distinctly not the case. Indeed, for the two-dimensional framework analyzed, the stall regime is not only more potent than the kinking regime, elevated measures of toughness exist over a broad range of grain angles prior to actual bridge formation.

The only cases which do not exhibit a stall regime correspond to $A R=2$, $\theta=87.5^{\circ}, 90^{\circ}$. In these specific cases, dynamics plays a role. Inertial effects are sufficient to suppress crack arrest and promote subsequent propagation up the far grain flank $\left(J^{k} \sim J^{b}\right)$.

To ensure the validity of these findings, a series of quasi-static simulations at $J^{s}$ were conducted. Prior findings were compared with pre-cracked geometries without cohesive surface elements. The resulting traction distributions ahead of the bridging grain are displayed in Figure 11 for $A R=3, \theta=60^{\circ}$. 
Note the unloading (dip) in the cohesive surface traction distribution at $J^{s}$ preceding reinitiation. The finite strength imposed by the cohesive surface model differentiates the prior solution from the pre-cracked geometry. An additional traction distribution is derived from the linear-elastic fracture mechanics (LEFM) solution is plotted for comparison. Pre-cracked geometries employing tip-tracking $a_{p}$ and a direct projection of the primary crack tip $a_{p p}$ yield identical traction distributions.

Simulations with and without cohesive surface elements differ near the origin of reinitiation but coincide at distances beyond $x \sim g_{l}$. Interestingly, both the cohesive and pre-crack simulations approach the LEFM solution in roughly $3 g_{l}$. We note that the radius of the disk upon which the $K$-field is applied is $\sim 111 g_{l}$. Although only one case is presented, other cases yielded similar findings. These findings confirm that the load for bridging is not sensitive to the method of crack tip projection, the compliance introduced by the cohesive surface formulation is negligible, and the nonlinear behavior near the crack tip is contained. The analog to the local, contained region is small-scale yielding.

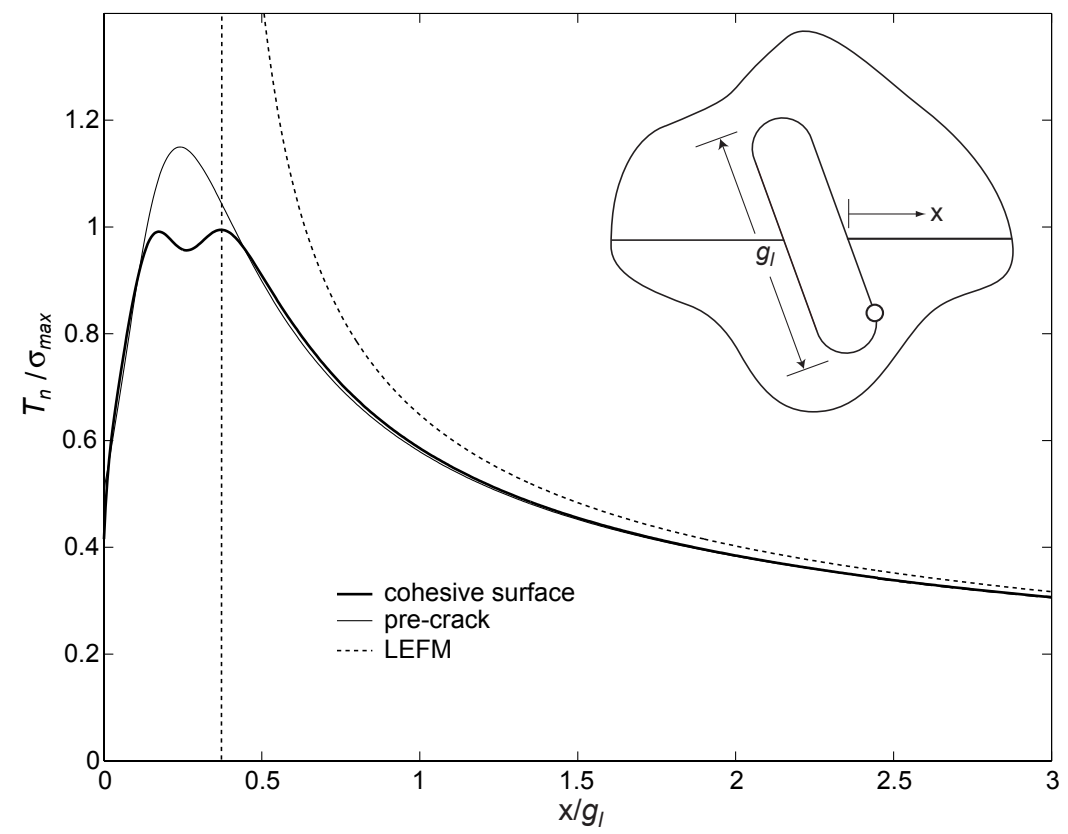

Fig. 11. Global traction distribution $T_{n}$ along the grain boundary ahead of the bridging grain for $A R=3, \theta=60^{\circ}$ and $J=J^{s}$. The compliance introduced by the cohesive surface formulation is negligible and the nonlinear behavior near the crack tip is contained. The linear elastic fracture mechanics (LEFM) solution is plotted for comparison.

Having established the validity of our findings, we construct a rationale for bridge formation. The basis for this construction lies in an idealization of the 
stall regime. The critical driving force for crack climbing can be correlated with kinking at $180^{\circ}-\theta$. Figure 7 illustrates that the load required to kink "backwards" $\left(\theta>90^{\circ}\right)$ rapidly increases with kink angle. Climb (kinking backwards) is a nonlinear process that varies with local crack configuration, grain length, and the number of active crack tips. Supplementing previous findings (Figure 10), we can calculate the critical driving force for the crack to climb $J^{b, c}$ and reinitiate $J^{b, r}$ at each aspect ratio and grain angle. The critical load for bridge formation $J^{b}$ is $\min \left(J^{b, c}, J^{b, r}\right)$. As the driving force required to climb rapidly increases, reinitiation becomes energetically favorable.

Curves of normalized, critical driving force for climb and reinitiation are drawn in Figure 12. Because each case yields either climb or reinitiation, additional simulations are conducted to "force" climb or reinitiation. Specifically, the cohesive surface elements on the grain boundary along the grain flank (forced reinitiation) or ahead of the primary crack tip (forced climb) are removed. Figure 12 overlays results from Figure 7 and the additional, "forced" simulations on Figure 10. Bounds are given for forced reinitiation because the load required to reinitiate is sensitive to crack tip location. Crack tip locations of the primary crack are taken at instability $J^{s}$. The lower bound, (-), and upper bound, $(+)$, derive from locations of the peak $\left(T_{n} \sim \sigma_{\max }\right)$ and tail $\left(T_{n} \sim 0.1 \sigma_{\max }\right)$ of the cohesive zone, respectively. The additional forced cases confirm the con-

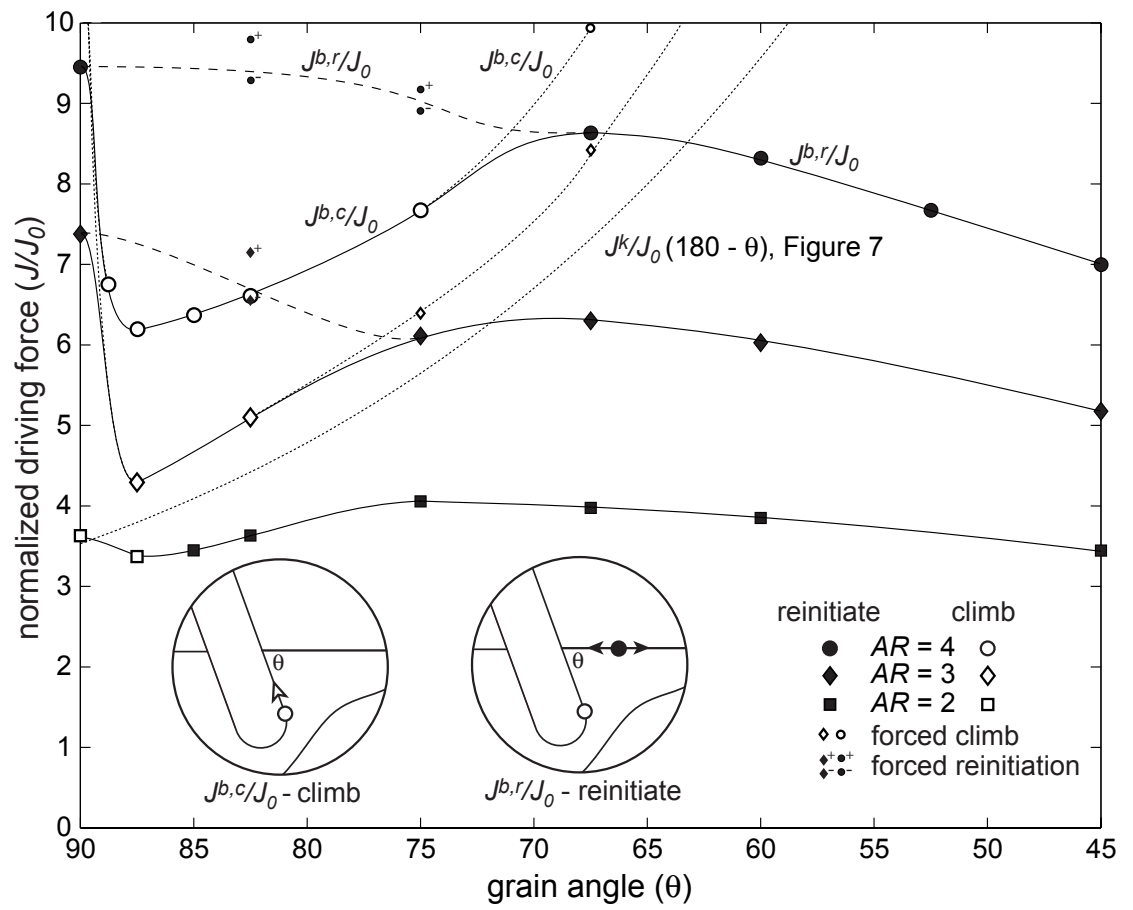

Fig. 12. The critical normalized driving force for bridging $J^{b} / J_{0}$ is the minimum of the normalized driving force to climb $J^{b, c} / J_{0}$ or reinitiate $J^{b, r} / J_{0}$. Dotted curves represent forced climb while dashed curves signify forced reinitiation. 
dition for bridge formation, $\min \left(J^{b, c}, J^{b, r}\right)$. As the driving force required to climb rapidly increases, reinitiation ahead of the primary tip is energetically favorable. The forced curves illustrate that the local maximum near $\theta=68^{\circ}$ for $A R=3$ and $A R=4$ correlates with a change in mechanism, climb $\rightarrow$ reinitiation. Likewise, the rapid decline in the resistance near $\theta=90^{\circ}$ for the same aspect ratios can also the correlated with a change in mechanism, reinitiation $\rightarrow$ climb. The symmetry of the $\theta=90^{\circ}$ configuration enables the two crack tips to strongly interact (through repulsion). In only a degree, the symmetry is sufficiently broken and a single crack climbs in a manner consistent with Figure 7. Figure 13 highlights the rapid decrease in resistance for $A R=4$. Crack reinitiation ahead $\left(\theta=90^{\circ}\right)$ or climb along the far flank $\left(\theta=87.5^{\circ}\right)$ results in a toughness of $J^{b} / J_{0}=9.45$ or $J^{b} / J_{0}=6.14$, respectively.

Although we have presented findings for the evolution of the bridge after climb or reinitiation, we hesitate to assign particular importance to the details of crack propagation after $J^{b}$. While the paths of propagation are physically reasonable, the single, overdriven 2-D bridge cannot shed load to evolve neighboring bridges. Furthermore, we do not currently employ grain boundary friction. We only enforce interpenetrability. Although friction and additional obstacles (bridges) can be incorporated into the current framework, the current work examines the evolution of a single bridge and make no attempt to resolve the bridging zone.

We also note that the current framework does not presently consider transgranular fracture. Depending on the grain aspect ratio and grain angle, small volumes of microstructure in the kink and/or stall regimes are subjected to principal stresses ranging from $E / 12$ to $E / 6$. Because these values approach the theoretical strength of the grain, future work will address transgranular fracture and the role of both grain and grain boundary material properties in the bridging process.

\subsection{Application to structural ceramics}

Although grain bridging is a well documented mechanism for the toughness of structural ceramics, the present model identifies a significant toughening effect even before the bridge is fully formed. As such toughening is induced within a few grains of the crack tip, this mechanism provides for a rapid initial rise in the R-curve (a desirable characteristic for strength in the presence of small flaws), and (as noted) for the initiation of cracks ahead of the primary crack tip. The latter phenomenon is interesting as this is quite unexpected for a material with limited inelasticity. In ductile (metallic) materials, it is

well known that due to crack-tip blunting, cracks can readily initiate ahead of the primary crack tip as the maximum local stresses peak there (typically on 


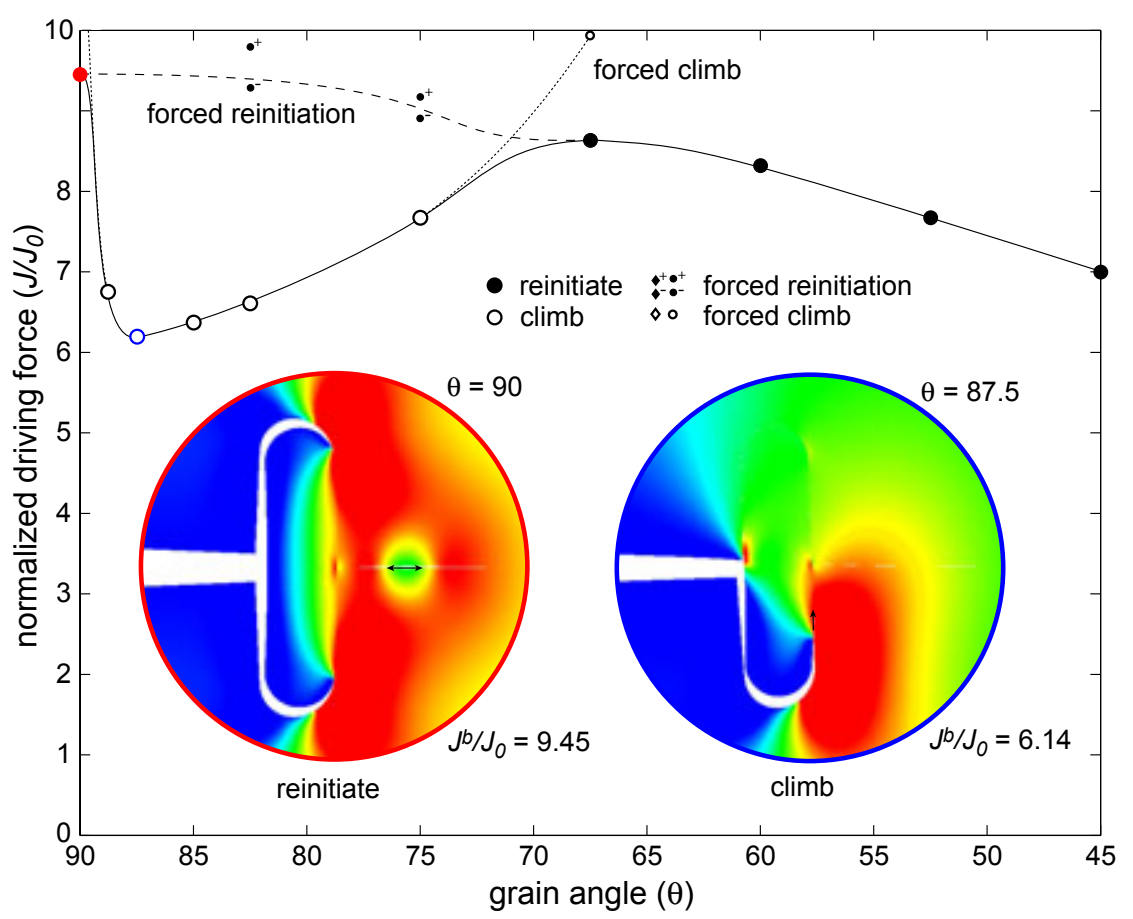

Fig. 13. The critical normalized driving force for bridging $J^{b} / J_{0}$ for $A R=4$. The $\sigma_{22}$ stress field is plotted for the global maximum, $\theta=90^{\circ}$, and a local minima, $\theta=87.5^{\circ}$. Contour levels mirror Figure 9.

the order of two crack tip opening displacements ahead of the tip); in brittle (ceramic) materials, conversely, blunting is effectively non-existent and the peak local stresses and corresponding sites for crack initiation are restrained to the immediate vicinity of the crack tip. One of the consequences of such crack initiation ahead of the primary crack tip is that the imperfect linking of initiated cracks to the primary crack can result in the formation of uncracked regions in the wake of the new crack tip, which can then act as bridges as the crack opens - so called "uncracked ligament bridging" (Shang and Ritchie, 1989). This form of bridging has been reported for $\mathrm{SiC}$, but it has not been known until now how such bridges are able to form.

Despite the idealized nature of this model, the reported predictions are in accord with general experimental observations. Numerous micrographs published for in situ toughened $\mathrm{Si}_{3} \mathrm{~N}_{4}$ and $\mathrm{SiC}$ ceramics with bimodal grain size distributions illustrate bridged grains consistent with current findings. Figure 14 is representative of the literature. Note that a portion of the inclined, bridged grain remains intact while the remaining portion is debonded. Current findings indicate that for $A R=4, \theta=60^{\circ}$ the peak driving force and corresponding stress intensity for bridge formation are significant, $8.6 J_{b}$ and $2.9 K_{b}$, respectively.

Although we acknowledge that bridge formation is a 3-D process subject to 


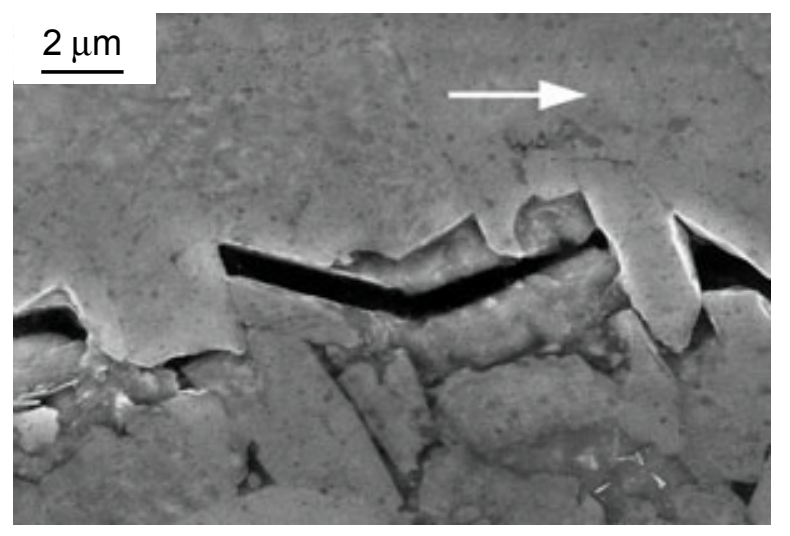

Fig. 14. Fractographic sections showing grain bridging through the development of interlocking grains in silicon carbide. The horizontal arrow shows the general direction of crack propagation.

variations in geometry and material properties, we submit that the idealized 2-D framework is especially applicable to the interlocking, plate-like grains of $\mathrm{SiC}$ (Gilbert et al., 1996). The analysis must be extended to 3-D to determine if a crack reinitiates ahead of the primary crack front or if the crack front merely "wraps" around the inclined grain. Depending on the grain shape, size, and orientation, one may envision a kink, arrest, and stall regime in mode I/II, mode I/III, or some combination thereof.

Often, cracks kink and transition from intergranular to transgranular fracture in one to two grain diameters $\left(1<g_{w}<2 \rightarrow 2<A R<4\right)$. After propagating through the elongated grain, the crack is essentially "stalled." If we now consider another transition to intergranular fracture, calculations indicate that load required for reinitiation can be substantial. We repeat that this mechanism is operable prior to bridge formation and thus may contribute to a rapid rise in the R-curve. While sufficiently steep R-curves have been recently noted in equiaxed alumina (Kruzic et al., 2005a), initial stages (one to two grain diameters) of the $\mathrm{R}$-curve in $\mathrm{Si}_{3} \mathrm{~N}_{4}$ and $\mathrm{SiC}$ have not been reported.

\section{Conclusion}

High strength in ceramic materials can be achieved at realistic flaw sizes through the development of a rapidly rising R-curve. In most monolithic structural ceramics, such R-curve toughening has been considered to arise from grain bridging. We propose a partition of the grain bridging process into five regimes: propagate, kink, arrest, stall, and bridge (Figure 4). Although the kinking regime (crack deflection) is an established toughening mechanism, the stall regime has not been investigated prior to this work. During this regime, 
continued propagation along the grain boundary will result in kinking backwards. The crack is essentially "stalled" and resists substantial crack-driving forces. Continued loading exposes large volumes of microstructure to stresses in excess of the grain boundary strength. For a broad range of grain inclination angles, cracks reinitiate ahead of the primary crack tip. Although reinitiation is not generally accepted for brittle materials, current findings indicate that reinitiation is energetically favorable to crack climbing. The driving force required to reinitiate is a substantially greater than the driving force required to kink. The stall mechanism is relatively insensitive to grain angle, operable prior to bridge formation, and may provide a rationale for a rapidly rising R-curve.

The proposed partition stems from an idealized two-dimensional framework of a single, non-equiaxed, inclined grain spanning two adjacent grains. Detailed studies at the microstructural level are performed with relatively simple grain (bulk) and grain boundary (surface) models. Cohesive surface elements enable crack initiation and propagation along grain boundaries. As modeling high-strength, low-toughness (brittle) systems requires substantial levels of discretization, $K$-field boundary conditions are applied to a $50 \mu \mathrm{m}$ disk containing $2.5 \mathrm{~nm}$ cohesive surface elements. Unstable propagation during quasi-static simulations requires implicit dynamics to yield a branch of the solution. The concatenation of quasi-static and dynamic simulations yields the evolution of grain bridging.

The main implications of this study are that significant R-curve toughening, associated with the stall regime, can be achieved in ceramic materials within a few grains of the crack tip, prior to actual grain bridging, and that crack reinitiation, ahead of the stalled, primary crack tip, is energetically favorable to continued propagation.

\section{Acknowledgements}

J.W.F. is grateful for the support of Sandia National Laboratories, operated by Sandia Corporation, a Lockheed Martin Company, for the United States Department of Energy under contract DE-AC04-94AL85000. R.O.R. acknowledges the support provided by the Director, Office of Science, Office of Basic Energy Sciences, Division of Materials Sciences and Engineering of the U.S. Department of Energy under Contract No. DE-AC02-05CH11231. This paper is dedicated to the memory of one of the authors, Dr. Rowland Cannon, who died suddenly and unexpectedly on April 21, 2006. 


\section{References}

Barenblatt, G.I., 1962. The mathematical theory of equilibrium cracks in brittle fracture. Advances in Applied Mechanics 7, 55-129.

Becher, P.F, 1991. Microstructural design of toughened ceramics. Journal of the American Ceramic Society 74, 255-269.

Becher, P.F., Hsueh, C.-H., Alexander, K.B., Sun, E.Y., 1996. Influence of reinforcement content and diameter on the $\mathrm{R}$-curve response in $\mathrm{SiC}$-whiskerreinforced alumina. Journal of the American Ceramic Society 79, 298-304.

Becher, P.F., Sun, E.Y., Plucknett, K.P., Alexander, K.B., Hsueh, C.-H., Lin, H.-T., Waters, S.B., Westmoreland, C.G., 1998. Microstructural design of silicon nitride with improved fracture toughness: I, effect of grain size and shape. Journal of the American Ceramic Society 81, 2821-2830.

Bennison, S.J., Lawn, B.R., 1989. Role of interfacial grain-bridging sliding friction in the crack-resistance and strength properties of nontransforming ceramics. Acta Metallurgica 37, 2659-2671.

Bilby, B.A., Cardew, G.E., Howard, I.C., 1977. Stress intensity factors at the tips of kinked and forked cracks. In: Taplin, D. (Ed.), Fracture 1977 - Advances in research on the strength and fracture of materials. Vol. 3. Pergamon Press, New York, pp. 197-200.

Camacho, G.T., Ortiz, M., 1996. Computational modeling of impact damage in brittle materials. International Journal of Solids and Structures 33, 28992938.

Cao, J.J., MoberlyChan, W.J., DeJonghe, L.C., Gilbert, C.J., Ritchie, R.O., 1996. In situ toughened silicon carbide with Al-B-C additions. Journal of the American Ceramic Society 79, 461-469.

Chantikul, P., Bennison, S.J., Lawn, B.R., 1990. Role of grain size in the strength and R-curve properties of alumina. The Journal of the American Ceramic Society 73, 2419-2427.

Cotterell, B., Rice, J.R., 1980. Slightly curved or kinked cracks. International Journal of Fracture 16, 155-169.

Davidge, R.W., Tappin, G., 1968. The effective surface energy of brittle materials. Journal of Materials Science 3, 165-173.

Eshelby, J.D., 1951. The force on an elastic singularity. Philosophical Transactions of the Royal Society of London A244, 87-112.

Espinosa, H.D., Zavattieri, P.D., 2003a. A grain level model for the study of failure initiation and evolution in polycrystalline brittle materials. part I: theory and numerical implementation. Mechanics of Materials 35, 323-364.

Espinosa, H.D., Zavattieri, P.D., 2003b. A grain level model for the study of failure initiation and evolution in polycrystalline brittle materials. part II: numerical examples. Mechanics of Materials 35, 365-394.

Evans, A.G., 1989. High toughness ceramics. Materials Science and Engineering A A105-106, 65-75.

Evans, A.G., 1990. Perspective on the development of high-toughness ceramics. Journal of the American Ceramic Society 73, 187-206. 
Evans, A.G., Tappin, G., 1972. Effect of microstructure on the stress to propagate inherent flaws. Proceedings of the British Ceramic Society 20, 275-297.

Faber, K.T., Evans, A.G., 1983. Crack deflection processes. I. theory. Acta Metallurgica 31, 577-584.

Gilbert, C.J., Cao, J.J., MoberlyChan, W.J., De Jonghe, L.C., Ritchie, R.O., 1996. Cyclic fatigue and resistance-curve behavior of an in situ toughened silicon carbide with Al-B-C additions. Acta Materialia 44, 3199-3214.

Guo, Z.K., Kobayashi, A.S., Hay, J.C., White, K.W., 1999. Fracture process zone modeling of monolithic $\mathrm{A}_{2} \mathrm{O}_{3}$. Engineering Fracture Mechanics 63, 115129.

Hay, J.C., White, K.W., 1993. Grain-bridging mechanisms in monolithic alumina and spinel. Journal of the American Ceramic Society 76, 1849-54.

He, M.-Y., Hutchinson, J.W., 1989. Kinking of a crack out of an interface. Journal of Applied Mechanics 56, 270-278.

Helms, K.L.E., Allen, D.H., Hurtado, L.D., 1999. A model for predicting grain boundary cracking in polycrystalline viscoplastic materials including scale effects. International Journal of Fracture 95, 175-194.

Hübner, H., Jillek, R.W., 1977. Sub-critical crack extension and crack resistance in polycrystalline alumina. Journal of the American Ceramic Society $76,1849-54$.

Hutchinson, J.W., Suo, Z., 1991. Mixed mode cracking in layered materials. Advances in Applied Mechanics 29, 63-187.

Klein, P.A., Foulk, J.W., Chen, E.P., Wimmer, S.A., Gao, H.J., 2001. Physicsbased modeling of brittle fracture: cohesive formulations and the application of meshfree methods. Theoretical and Applied Fracture Mechanics 37, 99166.

Knehans, R., Steinbrech, R., 1982. Memory effect of crack resistance during slow crack growth in notched $\mathrm{Al}_{2} \mathrm{O}_{3}$. Journal of Materials Science Letters 1, 327-329.

Kovalev, S., Miyajima, T., Yamauchi, Y., 1999. Micromechanics of crack bridging by elongated crystals in self-reinforced ceramic materials. Key Engineeering Materials 161-163, 615-618.

Kovalev, S., Miyajima, T., Yamauchi, Y., Sakai, M., 2000. Numerical evaluation of toughening by crack-face grain interlocking in self-reinforced ceramics. Journal of the American Ceramic Society 83, 817-824.

Kruzic, J.J., Cannon, R.M., Ager III, J.W., Ritchie, R.O., 2005a. Fatigue threshold r-curves for predicting reliability of ceramics under cyclic loading. Acta Materialia 53, 2595-2605.

Kruzic, J.J., Cannon, R.M., Ritchie, R.O., 2004. Crack-size effects on cyclic and monotonic crack growth in polycrystalline alumina: quantification of the role of grain bridging. Journal of the American Ceramic Society 87, 93-103.

Kruzic, J.J., Cannon, R.M., Ritchie, R.O., 2005b. Effects of moisture on the grain boundary strength, fracture, and fatigue properties of alumina. Journal of the American Ceramic Society 88, 2236-2245. 
Lange, F.F., 1973. Relation between strength, fracture energy and microstructure of hot-pressed $\mathrm{Si}_{3} \mathrm{~N}_{4}$. Journal of the American Ceramic Society 56, $518-522$.

Lathabai, S., Lawn, B.R., 1989. Fatigue limits in noncyclic loading of ceramics with crack-resistance curves. Journal of Materials Science 24, 4298-4306.

Lee, D.-H., Kim, H.-E., Cho, S.-J., 1994a. Microstructure and fracture toughness of hot-pressed $\mathrm{SiC}$ reinforced with $\mathrm{SiC}$ whisker. Journal of the American Ceramic Society 77, 3270-3272.

Lee, S.K., Kim, D.K., Kim, C.H., 1994b. Microstructure development and mechanical properties of pressureless-sintered $\mathrm{SiC}$ with plate-like grains using $\mathrm{Al}_{2} \mathrm{O}_{3}-\mathrm{Y}_{2} \mathrm{O}_{3}$ additives. Journal of Materials Science 29, 5321-5326.

Li, C.-W., Lee, D.J., Lui, S.C., 1992. R-curve behavior and strength for insitu reinforce silicon nitrides with different microstructures. Journal of the American Ceramic Society 75, 1777-1785.

Lo, K.K., 1978. Analysis of branched cracks. Journal of Applied Mechanics $45,797-802$.

Macmillan, N.H., 1972. Review: The theoretical strength of solids. Journal of Materials Science 7, 239-254

Mai, Y.-W., Lawn, B.R., 1987. Crack-interface grain bridging as a fracture resistance mechanism in ceramics: II, theoretical fracture mechanics model. Journal of the American Ceramic Society 70, 289-294.

Maiti, S., Geubelle, P.H., 2004. Mesoscale modeling of dynamic fracture of ceramic materials. Computer Modeling in Engineering and Sciences 5, 91101.

Marshall, D.B., 1988. Interfaces and toughening in ceramics. Journal de Physique Colloque 49, 25-33.

Marshall, D.B., Evans, A.G., 1988. The influence of residual stress on the toughness of reinforced brittle materials. Materials Forum 11, 304-312.

MoberlyChan, W.J., DeJonghe, L.C., 1998. Controlling interface chemistry and structure to process and toughen silicon carbide. Acta Materialia 46, $2471-2477$.

Needleman, A., 1987. A continuum model for void nucleation by inclusion debonding. Journal of Applied Mechanics 54, 525-531.

Needleman, A., 1990. An analysis of tensile decohesion along an interface. Journal of the Mechanics and Physics of Solids 38, 289-324.

Padture, N.P., 1994. In situ toughened silicon carbide. Journal of the American Ceramic Society 77, 519-523.

Rice, J.R., 1968. A path independent integral and the approximate analysis of stress concentration by notches and cracks. Journal of the Mechanics and Physics of Solids 40, 939-963.

Ritchie, R.O., Gilbert, C.J., McNaney, J.M., 2000. Mechanics and mechanisms of fatigue damage and crack growth in advanced materials. International Journal of Solids and Structures 73, 311-329.

Rödel, J., Kelly, J.F., Lawn, B.R., 1990. In situ measurements of bridged crack interfaces in the scanning electron microscope. Journal of the American 
Ceramic Society 73, 3313-3318.

Sajgalik, P., Dusza, J., Hoffmann, M.J., 1995. Relationship between microstructure, toughening mechanisms, and fracture toughness of reinforced silicon nitride ceramics. Jounal of the American Ceramic Society 78, 26192624 .

Satet, R.L., Hoffmann, M.J., Cannon, R.M., 2006. Experimental evidence of the impact of rare-earth elements on particle growth and mechanical behavior of silicon nitride. Materials Science and Engineering A 422, 66-76.

Shang, J.K., Ritchie, R.O., 1989. Crack bridging by uncracked ligaments during fatigue-crack growth in SiC-reinforced aluminum-alloy composites. Metallurgical Transactions A 20A, 897-908.

Simo, J.C., Hughes, T.J.R., 1997. Computational Inelasticity. Springer-Verlag, New York.

Simo, J., Taylor, R., Pister, K., 1985. Variational and projection methods for the volume constraint in finite deformation elastoplasticity. Computer Methods in Applied Mechanics and Engineering 51, 177-208.

Steinbrech, R., Knehans, R., Schaarwächter, W., 1983. Increase of crack resistance during slow crack growth in $\mathrm{Al}_{2} \mathrm{O}_{3}$ bend specimens. Journal of Materials Science 18, 265-270.

Swain, M.V., 1986. R-curve behavior in a polycrystalline alumina material. Journal of Materials Science Letters 5, 1313-1315.

Swanson, P.L., Fairbanks, C.J., Lawn, B.R., Mai, Y.-W., Hickey, B.J., 1987. Crack-interface grain bridging as a fracture resistance mechanicsm in ceramics: I, experimental study on alumina. Journal of the American Ceramic Society 70, 279-289.

Thouless, M.D., Sbaizero, O., Sigl, L.S., Evans, A.G., 1989. Effect of interface mechanical properties on pullout in a SiC-fiber-reinforced lithium aluminum silicate glass-ceramic. Journal of the American Ceramic Society 72, 525-532.

Tran, D.K., Kobayashi, A.S., White, K.W., 2001. Crack growth in alumina at high temperature. Engineering Fracture Mechanics 68, 149-161.

Tvergaard, V., Hutchinson, J.W., 1990. Effect of fiber debonding in a whiskerreinforced metal. Materials Science and Engineering A125, 203-213.

Tvergaard, V., Hutchinson, J.W., 1992. The relation between crack growth resistance and fracture process parameters in elastic-plastic solids. Journal of the Mechanics and Physics of Solids 40, 1377-1397.

Van Weeren, R., Danforth, S.C., 1996. The effect of grain boundary phase characteristics on the crack deflection behavior in a silicon nitride material. Scripta Materialia 384, 1567-1573.

Vekinis, G., Ashby, M.F., Beumont, P.W.R., 1990. R-curve behavior of $\mathrm{Al}_{2} \mathrm{O}_{3}$ ceramics. Acta Materialia 38, 1151-1162.

Willis, J.R., 1967. A comparison of the fracture criteria of Griffith and Barenblatt. Journal of the Mechanics and Physics of Solids 15, 151-162.

Xu, H.H.K., Wei, L., Pature, N.P., Lawn, B.R., Yeckley, R.L., 1995. Effect of microstructural coarsening on short-crack toughness properties of silicon nitride. Journal of Materials Science 30, 869-878. 
Xu, X.-P., Needleman, A., 1994. Numerical simulations of fast crack growth in brittle solids. Journal of the Mechanics and Physics of Solids 42, 1397-1434.

Zavattieri, P.D., Espinosa, H.D., 2001. Grain level analysis of crack initiation and propagation in brittle materials. Acta Materialia 49, 4291-4311.

Zavattieri, P.D., Espinosa, H.D., 2003. An examination of the competition between bulk behavior and interfacial behavior of ceramics subjected to dynamic pressure-shear loading. Journal of the Mechanics and Physics of Solids 51, 607-635.

Zavattieri, P.D., Raghuram, P.V., Espinosa, H.D., 2001. A computational model of ceramic microstructures subjected to multi-axial dynamic loading. Journal of the Mechanics and Physics of Solids 49, 27-68. 